\title{
Constraints on a massive double-copy and applications to massive gravity
}

\author{
Laura A. Johnson, ${ }^{a}$ Callum R. T. Jones ${ }^{b}$ and Shruti Paranjape ${ }^{b}$ \\ ${ }^{a}$ CERCA, Department of Physics, Case Western Reserve University, \\ 10900 Euclid Ave, Cleveland, OH 44106, U.S.A. \\ ${ }^{b}$ Leinweber Center for Theoretical Physics, Randall Laboratory of Physics, \\ The University of Michigan, \\ Ann Arbor, MI 48109-1040, U.S.A. \\ E-mail: laura.johnson2@case.edu, jonescal@umich.edu, shrpar@umich.edu
}

ABSTRACT: We propose and study a BCJ double-copy of massive particles, showing that it is equivalent to a KLT formula with a kernel given by the inverse of a matrix of massive bi-adjoint scalar amplitudes. For models with a uniform non-zero mass spectrum we demonstrate that the resulting double-copy factors on physical poles and that up to at least 5-particle scattering, color-kinematics duality satisfying numerators always exist. For the scattering of 5 or more particles, the procedure generically introduces spurious singularities that must be cancelled by imposing additional constraints. When massive particles are present, color-kinematics duality is not enough to guarantee a physical double-copy. As an example, we apply the formalism to massive Yang-Mills and show that up to 4-particle scattering the double-copy construction generates physical amplitudes of a model of dRGT massive gravity coupled to a dilaton and a two-form with dilaton parity violating couplings. We show that the spurious singularities in the 5-particle double-copy do not cancel in this example, and the construction fails to generate physically sensible amplitudes. We conjecture sufficient constraints on the mass spectrum, which in addition to massive BCJ relations, guarantee the absence of spurious singularities.

KEYwords: Scattering Amplitudes, Classical Theories of Gravity

ArXiv EPrint: 2004.12948 


\section{Contents}

1 Introduction 1

2 Massive KLT formula $\quad 7$

2.1 Equivalence of massive BCJ and massive KLT 7

$\begin{array}{lll}2.2 & \text { Spurious singularities } & 11\end{array}$

3 Massive gravity and (Massive Yang-Mills) ${ }^{2} \quad 13$

$\begin{array}{lll}3.1 & \text { Physical motivation } & 14\end{array}$

$\begin{array}{lll}3.2 & 3 \text {-point amplitudes and asymptotic states } & 16\end{array}$

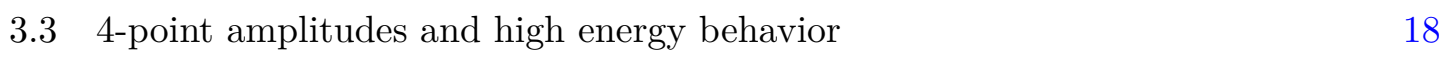

$\begin{array}{lll}3.4 & 5 \text {-point amplitudes and non-physical singularities } & 22\end{array}$

4 Locality and the spectral condition $\quad 23$

4.1 4-point spectral condition 24

$\begin{array}{lll}4.2 & 5 \text {-point spectral conditions } & 29\end{array}$

4.3 Non-minimal rank 32

5 Discussion $\quad 32$

A Massless limits of massive theories $\quad 34$

B Matrix of 5-point bi-adjoint scalar amplitudes $\quad 38$

$\begin{array}{ll}\text { C Factorization on physical poles } & 40\end{array}$

D Feynman rules for massive Yang-Mills $\quad 44$

E 4-point graviton-dilaton amplitudes from double-copy $\quad 44$

$\begin{array}{lll}\text { F } & \text { BCJ relations as null vectors } & 46\end{array}$

\section{Introduction}

The essence of the double-copy is the existence (or conjectured existence) of a map from the physical observables $\mathcal{O}$ of a pair of models $A$ and $B$, each with a non-Abelian internal symmetry structure, to physical observables in some other model $A \otimes B$, without such a symmetry

$$
\mathcal{O}_{A} \times \mathcal{O}_{B} \mapsto \mathcal{O}_{A \otimes B}
$$

The original and best-studied example of such a map is given by the construction of KawaiLewellen-Tye (KLT), relating tree-level open and closed string scattering amplitudes [1], 
and the associated field theory limit $\left(\alpha^{\prime} \rightarrow 0\right)$ relating Yang-Mills and Einstein gravity. For example at 4-point

$$
\mathcal{M}_{4}^{\text {Grav }}(1,2,3,4)=-s_{14} \mathcal{A}_{4}^{\mathrm{YM}}[1,2,3,4] \mathcal{A}_{4}^{\mathrm{YM}}[1,3,2,4] .
$$

The double-copy has subsequently been extended to non-Abelian gauge theories with matter fields in non-adjoint representations $[2,3]$, to non-linear sigma models and D-brane worldvolume EFTs [4], generalized to loop-level [5] and even extended to classes of classical solutions [6]. See [7] and references therein for a comprehensive review of recent developments. More than a theoretical curiosity, there are often significant practical advantages to making use of such a map whenever it is available. Recent use of a generalized double-copy construction for Feynman integrands in $\mathcal{N}=8$ supergravity allowed the first explicit calculation of 4-point, 5-loop scattering amplitudes [8], a feat that is practically impossible to replicate by other, presently available means.

It is therefore a timely and relevant theoretical problem to understand the potential scope for generalizing the double-copy, and demarcating the boundary between those models which admit a double-copy structure and those which do not. In this paper we will be concerned with the problem of generalizing the field theory double-copy relation for treelevel scattering amplitudes to models with massive particles in the spectrum. Our central result is the demonstration that, when massive particles are present, color-kinematics $d u$ ality is not enough to guarantee a physically well-defined double-copy. We present in detail an explicit example, massive Yang-Mills, for which color-kinematics duality satisfying numerators exist (up to at least $n=5$, where $n$ is the number of external partucles), but for which the BCJ double-copy prescription generates expressions with non-physical spurious singularities.

To understand the generalization we propose in this paper, it is useful to first review the well-known construction of the double-copy for tree-level scattering amplitudes in pure Yang-Mills first described by Bern, Carrasco and Johansson (BCJ) [9]. We begin by organizing tree-level scattering amplitudes as a sum over trivalent graphs ${ }^{1}$

$$
\mathcal{A}_{4}\left(1^{a_{1}}, 2^{a_{2}}, 3^{a_{3}}, 4^{a_{4}}\right)=\frac{c_{12} n_{12}}{s_{12}}+\frac{c_{13} n_{13}}{s_{13}}+\frac{c_{14} n_{14}}{s_{14}} .
$$

This form of the amplitude reveals the remarkable, hidden property of color-kinematics duality, the numerators satisfy a sum rule

$$
n_{12}+n_{13}+n_{14}=0
$$

mirroring the Jacobi relation of the color factors

$$
c_{12}+c_{13}+c_{14}=0 .
$$

Perhaps even more remarkably, making the replacement $c_{i} \rightarrow n_{i}$ gives an expression

$$
\mathcal{M}_{4}(1,2,3,4)=\frac{n_{12}^{2}}{s_{12}}+\frac{n_{13}^{2}}{s_{13}}+\frac{n_{14}^{2}}{s_{14}},
$$

\footnotetext{
${ }^{1}$ We will use the following convention $c_{12}=f^{a_{1} a_{2} b} f^{a_{3} a_{4} b}, c_{13}=f^{a_{1} a_{3} b} f^{a_{4} a_{2} b}$ and $c_{14}=f^{a_{1} a_{4} b} f^{a_{2} a_{3} b}$. We also use Mandelstam invariants with all outgoing momenta, i.e. $s_{i j}=\left(p_{i}+p_{j}\right)^{2}$.
} 
which coincides with a scattering amplitude in a model of Einstein gravity coupled to a massless dilaton and Kalb-Ramond two-form. At higher multiplicity $(n \geq 5)$ there will be multiple, independent color Jacobi relations, corresponding to various different choices of triples of trivalent graphs with related topology, the generalization of (1.4) being that the (signed) sum of the numerators of these triples must vanish. The fundamental result of BCJ was to prove this BCJ double-copy and the property of color-kinematics duality persist at all multiplicity [9].

The BCJ numerators given by the trivalent organization of the amplitude are nonunique; as a consequence of the color Jacobi relation (1.5), the amplitude (1.3) is unchanged by a so-called generalized gauge transformation. For example for $n=4$

$$
n_{12} \rightarrow n_{12}+s_{12} \Delta, \quad n_{13} \rightarrow n_{13}+s_{13} \Delta, \quad n_{14} \rightarrow n_{14}+s_{14} \Delta,
$$

where $\Delta$ is an arbitrary function of the Mandelstam invariants. The BCJ numerators given by constructing the amplitude using standard Feynman rules, in general, do not satisfy the kinematic Jacobi relations. The challenge in applying the double-copy is to find an appropriate set of generalized gauge transformations which produce numerators which do satisfy the kinematic Jacobi relation. However, there is no a priori guarantee that such a generalized gauge can be found. This can be illustrated in the simplest case at $n=4$, suppose the Feynman-rule constructed numerators satisfy

$$
n_{12}+n_{13}+n_{14}=\mathcal{E}
$$

for some $\mathcal{E}$. Then making a generalized gauge transformation

$$
n_{12}+n_{13}+n_{14} \rightarrow n_{12}+n_{13}+n_{14}+\Delta\left(s_{12}+s_{13}+s_{14}\right)=\mathcal{E},
$$

where we have used the kinematic identity $s_{12}+s_{13}+s_{14}=0$. We conclude that if $\mathcal{E} \neq 0$, then there exists an obstruction to finding a generalized gauge in which the numerators satisfy the kinematic Jacobi relation (1.4). As we will review in section 2, at all multiplicities such obstructions are absent only if the color-ordered partial amplitudes of the model satisfy an infinite set of (generalized) gauge-invariant identities known as the BCJ relations. Colorkinematics duality is therefore a special property enjoyed by some models and not others.

This statement can be clearly illustrated in the context of an explicit example, first described in [10]. Consider a model of a $U(N)$ Yang-Mills theory coupled to a massless, adjoint, Majorana fermion in $d$-dimensions. For $n=4$ scattering with four external fermions we find

$$
\mathcal{E} \propto\left(\gamma_{\mu}\right)_{a_{1} a_{2}}\left(\gamma^{\mu}\right)_{a_{3} a_{4}}+\left(\gamma_{\mu}\right)_{a_{2} a_{3}}\left(\gamma^{\mu}\right)_{a_{1} a_{4}}+\left(\gamma_{\mu}\right)_{a_{3} a_{1}}\left(\gamma^{\mu}\right)_{a_{2} a_{4}}
$$

where $\gamma_{\mu}$ form some representation of the $d$-dimensional Clifford algebra. This expression is zero only in dimensions $d=3,4,6$ and 10, and therefore only in those dimensions does the model described satisfy color-kinematics duality. Said another way, in $d \neq 3,4,6$ or 10 the scattering amplitudes are perfectly physical, but there is an obstruction to finding a generalized gauge in which the BCJ numerators satisfy the kinematic Jacobi relation, and consequently there is no well-defined notion of a double-copy. 
While beautifully simple, it is not at all obvious that expressions like (1.6), and more importantly its generalizations to higher multiplicity, are actually physical scattering amplitudes. In particular, this construction fails to manifest locality in the form of the absence of spurious, non-propagator-like, singularities and the factorization of amplitudes on propagator-like, physical singularities. For $n \geq 5$ the generalized gauge functions needed to bring the local form of BCJ numerators generated by Feynman rules, to a color-kinematics duality satisfying representation, can in principle be arbitrarily complicated, non-local functions. There is an indirect argument that the result of the double-copy should be an expression with the locality properties of a scattering amplitude. Here we must make two additional assumptions about the color structure: the gauge group is $U(N)$ and all of the external states are in the adjoint representation. ${ }^{2}$ This covers pure Yang-Mills and its supersymmetrizations, but excludes other known examples of the double-copy, such as QCD-like models with matter fields in the fundamental representation $[2,3]$. Throughout this paper we will always make these assumptions, leaving possible generalizations to future work. As shown explicitly in [9] it is possible to prove that, with these additional assumptions, the BCJ double-copy is equivalent to the KLT double-copy. By making a convenient choice of the basis of partial amplitudes in the KLT sum, this form of the double-copy manifests the absence of spurious singularities. ${ }^{3}$

Clearly however, the BCJ form of the double-copy (1.3) would manifest locality if we could find a generalized gauge in which all of the numerators are simultaneously local functions. While it may be an empirical fact that among the diverse range of color-kinematics duality compatible models, such local numerators can often be found, we are not aware of a general argument that this should always be possible. The existence of local numerators is then possibly a stronger assumption than color-kinematics duality, but at least for those models which admit a KLT representation of the double-copy, it is also an unnecessary assumption. The proof of the equivalence of the BCJ and KLT double-copies requires only that the numerators satisfy the kinematic Jacobi, and makes no assumption about the locality structure thereof; if duality satisfying numerators can only be found in a non-local form then this just means that any spurious singularities must cancel in the sum over trivalent graph contributions. In the context of the familiar massless double-copy we conclude that, in addition to the usual S-matrix axioms of locality, unitarity (factorization), Lorentz invariance, as well as the assumption that the model has the required color or flavor symmetry structure to admit a KLT form of the double-copy (1.3), the property of color-kinematics duality is a necessary and sufficient condition for the double-copy to be a physical scattering amplitude. One of the main results of this paper is an explicit demonstration that when, in addition to the above assumptions, massive states are present in the spectrum, color-kinematics duality is no longer a sufficient condition to avoid spurious, non-physical, singularities in the double-copy.

\footnotetext{
${ }^{2}$ One can also apply this argument to $S U(N)$ gauge groups, but here we need the additional assumption that there are no multi-trace contributions to tree-level scattering amplitudes. For simplicity, for the remainder of the paper we will assume that the gauge group is $U(N)$.

${ }^{3}$ Equation (1.2) illustrates the main idea. The only possible non-physical singularity that could appear in this expression is a possible doubling of the $s_{14}=0$ pole, but this is clearly removed by a corresponding zero in the KLT kernel.
} 
The BCJ construction has a natural extension to models containing massive states, for which various special cases have been considered previously [3, 11-20]. To our knowledge, no completely general description of a massive BCJ double-copy, and the associated constraints, has been given. In particular, the case of double-copying amplitudes in theories with no massless particles has not been studied before. This paper is a first step towards such a description, and an exploration of the various problems that may arise.

The direct analogue of the BCJ form of the amplitude for models with a uniform, non-zero mass spectrum is ${ }^{4}$

$$
\mathcal{A}_{4}^{m \neq 0}\left(1^{a_{1}}, 2^{a_{2}}, 3^{a_{3}}, 4^{a_{4}}\right)=\frac{c_{12} n_{12}}{s_{12}+m^{2}}+\frac{c_{13} n_{13}}{s_{13}+m^{2}}+\frac{c_{14} n_{14}}{s_{14}+m^{2}} .
$$

To construct the massive double-copy of such a model, we will follow closely the discussion above, and try to construct numerators which satisfy the kinematic Jacobi relation $n_{12}+$ $n_{13}+n_{14}=0$. If we succeed, we make the replacement $c_{i} \rightarrow n_{i}$ and construct the would-be massive double-copy

$$
\mathcal{M}_{4}^{m \neq 0}(1,2,3,4)=\frac{n_{12}^{2}}{s_{12}+m^{2}}+\frac{n_{13}^{2}}{s_{13}+m^{2}}+\frac{n_{14}^{2}}{s_{14}+m^{2}} .
$$

The central problem in this paper will be to understand the conditions under which expressions such as (1.12) and its natural generalization to higher multiplicity, define physical scattering amplitudes. At this point we make a simple observation: if we suppose that a BCJ representation of our massive model is constructed, perhaps using Feynman rules, with numerators satisfying

$$
n_{12}+n_{13}+n_{14}=\mathcal{E}
$$

then by making the following generalized gauge transformation

$$
\begin{aligned}
n_{12} \rightarrow \hat{n}_{12} & \equiv n_{12}+\frac{1}{m^{2}}\left(s_{12}+m^{2}\right) \mathcal{E} \\
n_{13} \rightarrow \hat{n}_{13} & \equiv n_{13}+\frac{1}{m^{2}}\left(s_{13}+m^{2}\right) \mathcal{E} \\
n_{14} \rightarrow \hat{n}_{14} & \equiv n_{14}+\frac{1}{m^{2}}\left(s_{14}+m^{2}\right) \mathcal{E},
\end{aligned}
$$

the amplitude (1.11) is invariant but the transformed numerators satisfy

$$
\hat{n}_{12}+\hat{n}_{13}+\hat{n}_{14}=0
$$

This generalized gauge transformation is well-defined for all $m \neq 0$ and all $\mathcal{E}$, so we can always find a generalized gauge that realizes color-kinematics duality! Since this argument relied only on knowledge of the spectrum, it applies independently of the details of the interactions. Contrary to the $m=0$ case where color-kinematics duality was a special property only found in a subset of models, usually with various special constraints on the spectrum of states and the associated interactions, for $m \neq 0$ it is no constraint at all. As we will see, this situation is indeed too good to be true. In section 2 we will rewrite the

\footnotetext{
${ }^{4}$ Throughout this paper we will use the mostly-plus metric convention $\eta_{\mu \nu}=\operatorname{diag}(-1,+1,+1,+1)$.
} 
would-be double-copy (1.12) in a KLT-like form with a kernel given by the inverse of a matrix of massive bi-adjoint scalar amplitudes [21, 22], we find that: $(i)$ the double-copy generically introduces non-local, spurious singularities for $n \geq 5$, and ( $i i)$ for $n=4$, gives a physical scattering amplitude, but fails to reduce to the standard double-copy in an appropriate $m \rightarrow 0$ decoupling limit.

These two major conclusions are not quite on equal footing. The result $(i)$ is fatal for any would-be double-copy with $n \geq 5$, it means that the result of applying the proposed massive generalization of the BCJ double-copy is an expression that could not have been calculated as a tree amplitude of a local quantum field theory. In section 4 we will extend our analysis to allow for a more complicated spectrum of states with possibly different masses, and provide evidence that if, in addition to the assumptions enumerated above, the masses satisfy a certain quadratic constraint then the problems with violations of locality are removed. The result (ii) is interesting, but does not mean that the massive double-copy for $n=4$ is non-physical. That something dramatic happens as $m \rightarrow 0$ could have been anticipated from the fact that the generalized gauge transformation (1.14) is singular in this limit. In the double-copied expression these inverse powers of mass will appear as coupling constants multiplying certain higher-derivative interactions that diverge as $m \rightarrow 0$. There is nothing illegal about this, indeed as we review in appendix A, interactions involving massive particles with spin $\geq 1$ generically diverge in the massless limit as some inverse power of the mass. In such cases a non-singular massless limit may be defined as an appropriate double-scaling or decoupling limit in which the coupling constants of the model are chosen to vanish with an appropriate positive power of the mass. Result (ii) can then be more accurately stated as the observation that the decoupling limit does not commute with the massive double-copy. Interestingly, under the additional constraints on the spectrum postulated in section 4 to ameliorate the non-locality in $n \geq 5$ particle scattering, we find that the decoupling limits and the massive double-copy do commute.

As a theoretical laboratory for making explicit calculations, we consider the physically motivated example of a model of massive Yang-Mills. As we explain in detail in section 3.1, by considering the reduction to the familiar massless double-copy in the high-energy or Goldstone boson equivalence limit, there is a plausible expectation that massive Yang-Mills double copies to a model of de Rham-Gabadadze-Tolley or dRGT massive gravity [23] coupled to a massive dilaton and a massive two-form. The primary conclusion of the analysis of this example is that no miraculous cancellation of the spurious singularities takes place, and the proposed massive double-copy fails to generate physical scattering amplitudes for $n \geq 5$. We will conclude by revisiting the logic of the above argument in section 4 and demonstrate that if, in addition to color-kinematics duality, the spectrum of masses satisfies certain constraints, then a local massive double-copy does indeed exist.

An outline of this paper is as follows. In section 2 we show that the massive BCJ double-copy can be equivalently formulated as a KLT-like product with a kernel given by the inverse of a $(n-2) ! \times(n-2)$ ! matrix of massive bi-adjoint scalar amplitudes. Here we use the Del Duca-Dixon-Maltoni (DDM) basis [24]. It is show that the KLT product does not smoothly reduce to the massless KLT product as $m \rightarrow 0$. For $n \geq 5$ the massive KLT kernel is shown to contain spurious singularities that cannot be associated 
with a factorization channel for any physical state, indicating the failure of the would-be double-copy to correspond to a scattering amplitude in a local field theory. In section 3 we present our primary explicit example, the double-copy of a mass-deformed version of Yang-Mills. At $n=3$ and $n=4$, the double-copy gives physically sensible results that can be interpreted as scattering amplitudes of a model of dRGT massive gravity coupled to a dilaton and two-form with a $\Lambda_{3}$ cutoff scale. At $n=5$, we numerically evaluate the residue on the spurious singularities, and confirm that they are non-zero, demonstrating that the BCJ double-copy does not produce a physical scattering amplitude. In section 4 we consider models with a general spectrum of masses. We show that if a certain condition is imposed on the spectrum, then the rank of the bi-adjoint scalar matrix is reduced and implies massive versions of the fundamental BCJ relations. It is shown that if the rank is reduced to $(n-3)$ ! then the massive double-copy takes a manifestly local form which reduces smoothly to the massless double-copy. In section 5 we conclude and describe important future directions.

While this work was in its final stages, the preprint [25] by Momeni, Rumbutis and Tolley appeared with some overlapping results at 4-point level.

\section{Massive KLT formula}

Under the assumptions outlined in the Introduction $(U(N)$ symmetry, external states in the adjoint representation, color-kinematics duality and the usual S-matrix axioms) it is possible to rewrite the BCJ double-copy as a KLT formula. In section 2.1 we show how this well-known argument can be extended to the proposed massive double-copy. This provides us with a different representation of the would-be double-copy in which the analysis of the singularity structure is more transparent. In section 2.2 we show that, term-by-term, the massive KLT sum contains spurious singularities and argue that a miraculous cancellation would need to take place for the final expression to contain only physical singularities.

\subsection{Equivalence of massive BCJ and massive KLT}

For any model with $U(N)$ symmetry, with asymptotic states in the adjoint representation, there exists a convenient decomposition of tree-amplitudes into single trace or color-ordered partial amplitudes of the form

$$
\mathcal{A}_{n}\left(1^{a_{1}}, \ldots, n^{a_{n}}\right)=\sum_{\sigma \in S_{n-1}} \operatorname{Tr}\left[T^{a_{1}} T^{a_{\sigma(2)}} \ldots T^{a_{\sigma(n)}}\right] \mathcal{A}_{n}[1 \sigma(2) \ldots \sigma(n)] .
$$

Without any further assumptions, the resulting $(n-1)$ ! partial amplitudes are generically independent.

The existence of a BCJ representation of the form (1.3) requires us to make the somewhat artificial assumption that the only color tensors which appear in vertex functions are contractions of the $U(N)$ structure constants $f^{a b c}$, as is the case for example in Yang-Mills. Various generalizations of the BCJ double-copy relaxing this assumption have been considered in the literature [7], but in this paper we will analyze only this simple Yang-Mills-like case. 
Assuming that such a BCJ representation exists, then the number of linearly independent partial amplitudes can be shown to be reduced to at least $(n-2)$ !. This reduction is accomplished by an additional set of linear constraints, known as the Kleiss-Kuijf (KK) relations [26]. If we further assume that the model satisfies color-kinematics duality, then by following the kinematic Jacobi analogue of the construction of the Dixon-Del DucaMaltoni (DDM) basis [24], the number of linearly independent BCJ numerators is likewise seen to be $(n-2)$ !.

If both representations exist, then since there are equal numbers of BCJ numerators and partial amplitudes, we should be able to translate between them by a linear transformation. For example, for $n=4$ for some arbitrary choice of numerators and partial amplitudes

$$
\left(\begin{array}{l}
\mathcal{A}_{4}[1234] \\
\mathcal{A}_{4}[1324]
\end{array}\right)=\left(\begin{array}{cc}
\frac{1}{s_{12}+m^{2}}+\frac{1}{s_{14}+m^{2}} & \frac{1}{s_{14}+m^{2}} \\
-\frac{1}{s_{14}+m^{2}} & -\frac{1}{s_{13}+m^{2}}-\frac{1}{s_{14}+m^{2}}
\end{array}\right)\left(\begin{array}{l}
n_{12} \\
n_{13}
\end{array}\right) .
$$

If $m \neq 0$, then the propagator matrix has full-rank and so we can solve for the kinematic Jacobi-satisfying numerators

$$
\left(\begin{array}{l}
n_{12} \\
n_{13}
\end{array}\right)=\left(\begin{array}{cc}
\frac{1}{s_{12}+m^{2}}+\frac{1}{s_{14}+m^{2}} & \frac{1}{s_{14}+m^{2}} \\
-\frac{1}{s_{14}+m^{2}} & -\frac{1}{s_{13}+m^{2}}-\frac{1}{s_{14}+m^{2}}
\end{array}\right)^{-1}\left(\begin{array}{l}
\mathcal{A}_{4}[1234] \\
\mathcal{A}_{4}[1324]
\end{array}\right) .
$$

When $m=0$ however, the propagator matrix has rank 1, and no such inversion is possible. In this case, the massless propagator matrix has a null-vector, and so we can make the replacement

$$
\left(\begin{array}{l}
n_{12} \\
n_{13}
\end{array}\right) \rightarrow\left(\begin{array}{l}
\hat{n}_{12} \\
\hat{n}_{13}
\end{array}\right)=\left(\begin{array}{l}
n_{12} \\
n_{13}
\end{array}\right)+\Delta\left(\begin{array}{l}
s_{12} \\
s_{13}
\end{array}\right)
$$

for any function $\Delta$. The existence of such null-vectors is indicative of an important difference between the massive and massless cases. For $m \neq 0$ the construction of numerators satisfying the kinematic Jacobi relations requires a complete fixing of the generalized gauge freedom. For $m=0$, this requires only a partial fixing. We can use this residual freedom to impose the gauge-fixing conditions $\hat{n}_{13}=0$, and solve for $\hat{n}_{12}$. From (2.2) with $m=0$, we have two different expressions for $\hat{n}_{12}$ which must be equal, leading to the so-called fundamental BCJ identity

$$
s_{12} \mathcal{A}_{4}[1234]=s_{13} \mathcal{A}_{4}[1324] .
$$

In general, the BCJ identities reduce the number of linearly independent partial amplitudes to $(n-3)$ ! [21, 27]. We can run this argument in both directions, reaching the well-known conclusion that the fundamental BCJ relations are necessary and sufficient conditions for the existence of color-kinematics duality satisfying BCJ numerators. In the massive case, due to the absence any residual generalized gauge freedom, there is no analogue of these identities. This is another way of saying that the constraint of color-kinematics duality is trivialized for models with a uniform massive spectrum.

This analysis generalizes naturally to $n$-point. For a model with uniform mass spectrum and $m \neq 0$ there is a linear relation of the form (2.2) relating the $(n-2)$ ! DDM bases of partial amplitudes and the kinematic numerators with an $(n-2) ! \times(n-2) !$ propagator 
matrix. We believe that this matrix is always of full-rank, but do not have a proof of this fact. An explicit expression for the $6 \times 6$ massive propagator matrix at $n=5$ is given in appendix $\mathrm{B}$, from which the rank can be verified to be 6 .

We will now proceed to derive a KLT form of the double-copy for the $m \neq 0$ case. We first rewrite the BCJ double-copy (1.11) in matrix form

$$
\mathcal{A}_{4}^{A \otimes B}(1,2,3,4)=\left(\begin{array}{ll}
n_{12}^{A} & n_{13}^{A}
\end{array}\right)\left(\begin{array}{cc}
\frac{1}{s_{12}+m^{2}}+\frac{1}{s_{14}+m^{2}} & -\frac{1}{s_{14}+m^{2}} \\
-\frac{1}{s_{14}+m^{2}} & \frac{1}{s_{13}+m^{2}}+\frac{1}{s_{14}+m^{2}}
\end{array}\right)\left(\begin{array}{l}
n_{12}^{B} \\
n_{13}^{B}
\end{array}\right),
$$

where we have already used the assumed kinematic Jacobi relations to express $n_{14}=$ $-n_{12}-n_{13}$. Combining this with our solution for the numerators (2.3) gives

$$
\begin{aligned}
& \mathcal{A}_{4}^{A \otimes B}(1,2,3,4) \\
& =\left(\begin{array}{ll}
\mathcal{A}_{4}^{A}[1234] & \mathcal{A}_{4}^{A}[1324]
\end{array}\right)\left(\begin{array}{cc}
\frac{1}{s_{12}+m^{2}}+\frac{1}{s_{14}+m^{2}} & -\frac{1}{s_{14}+m^{2}} \\
-\frac{1}{s_{14}+m^{2}} & \frac{1}{s_{13}+m^{2}}+\frac{1}{s_{14}+m^{2}}
\end{array}\right)^{-1}\left(\begin{array}{l}
\mathcal{A}_{4}^{B}[1234] \\
\mathcal{A}_{4}^{B}[1324]
\end{array}\right),
\end{aligned}
$$

which is of KLT form, with the matrix in the middle acting as a massive KLT kernel.

A similar calculation can be performed at 5-point, both to calculate the 6 independent BCJ numerators from a DDM basis of 6 partial amplitudes and to calculate the 5-point KLT kernel. The details of this calculation are presented in appendix B.

While we are in principle finished, to illustrate the robustness of this proposed generalization of the double-copy, we will now derive the same formula through a different line of argument. Somewhat recently, the massless KLT kernel was understood to be the inverse of a $(n-3) ! \times(n-3)$ ! matrix of tree-level scattering amplitudes of the following $U(N) \times U(\tilde{N})$ invariant model of massless scalars transforming in the bi-adjoint representation $[21,22]$

$$
\mathcal{L}=-\frac{1}{2}\left(\partial_{\mu} \phi^{a a^{\prime}}\right)^{2}-g f^{a b c} \tilde{f}^{a^{\prime} b^{\prime} c^{\prime}} \phi^{a a^{\prime}} \phi^{b b^{\prime}} \phi^{c c^{\prime}} .
$$

These amplitudes admit a double color-ordering

$$
\mathcal{A}_{n}^{\phi^{3}}\left(1^{a_{1} a_{1}^{\prime}}, \ldots, n^{a_{n} a_{n}^{\prime}}\right)=\sum_{\alpha, \beta \in S_{n-1}} \operatorname{Tr}\left[T^{a_{1}} T^{a_{\alpha(2)}} \ldots T^{a_{\alpha(n)}}\right] \operatorname{Tr}\left[\tilde{T}^{a_{1}^{\prime}} \tilde{T}_{\beta(2)}^{a^{\prime}} \ldots \tilde{T}^{a_{\beta(n)}^{\prime}}\right] \mathcal{A}_{n}^{\phi^{3}}[\alpha \mid \beta] .
$$

The partial amplitudes $\mathcal{A}_{n}^{\phi^{3}}[\alpha \mid \beta]$ are indexed by two orderings and can be constructed efficiently via a simple diagrammatic procedure [22]. Regarding $\mathcal{A}_{n}^{\phi^{3}}[\alpha \mid \beta]$ as an $(n-1) ! \times$ $(n-1)$ ! matrix of all possible orderings not related by a cyclic permutation, it can be shown to have rank $(n-3)$ ! [21]. The null vectors correspond to separate row and column KK and BCJ relations. For example at 4-point

$$
s_{12} \mathcal{A}_{4}^{\phi^{3}}[1234 \mid 1234]=s_{13} \mathcal{A}_{4}^{\phi^{3}}[1324 \mid 1234] .
$$

The central result of [21] was to prove that a BCJ-independent $(n-3) ! \times(n-3)$ ! sub-matrix has full-rank, and moreover has an inverse which is precisely equal to the KLT kernel in the given BCJ basis. The massless KLT formula can then be succinctly formulated as

$$
\mathcal{A}_{n}^{A \otimes B}(1,2, \cdots, n)=\sum_{\alpha, \beta} \mathcal{A}_{n}^{A}[\alpha]\left(\mathcal{A}^{\phi^{3}}\right)^{-1}[\alpha \mid \beta] \mathcal{A}_{n}^{B}[\beta],
$$


where $\alpha$ and $\beta$ range over, possibly distinct, BCJ bases of orderings of length $(n-3)$ !. This suggests a second, a priori independent, massive generalization of the KLT formula. Let us now investigate what happens in a massive bi-adjoint scalar theory

$$
\mathcal{L}=-\frac{1}{2}\left(\partial_{\mu} \phi^{a a^{\prime}}\right)^{2}-\frac{1}{2} m^{2} \phi^{a a^{\prime}} \phi^{a a^{\prime}}-g f^{a b c} \tilde{f}^{a^{\prime} b^{\prime} c^{\prime}} \phi^{a a^{\prime}} \phi^{b b^{\prime}} \phi^{c c^{\prime}} .
$$

Amplitudes in the massive theory are constructed using the same diagrammatic rules used for the massless theory [22], but with the massless propagators replaced with their massive counterparts. For example,

$$
\begin{aligned}
\mathcal{A}_{4}^{\phi^{3}}[1234 \mid 1234] & =\frac{1}{s_{12}+m^{2}}+\frac{1}{s_{14}+m^{2}}, \\
\mathcal{A}_{4}^{\phi^{3}}[1234 \mid 1324] & =-\frac{1}{s_{14}+m^{2}} .
\end{aligned}
$$

The 5-point matrix of bi-adjoint scalar amplitudes can be found in appendix B. The primary difference between the massless and massive bi-adjoint scalar amplitudes is in the number of independent color-orderings. In the massive theory, DDM orderings are independent and the $(n-2) ! \times(n-2)$ ! matrix of bi-adjoint scalar amplitudes has full-rank. Since this matrix is invertible, there is a natural conjecture for a massive KLT formula. At 4-point this takes the explicit form

$$
\begin{aligned}
& \mathcal{A}_{4}^{A \otimes B}(1,2,3,4) \\
& =\left(\begin{array}{ll}
\mathcal{A}_{4}^{A}[1234] & \mathcal{A}_{4}^{A}[1324]
\end{array}\right)\left(\begin{array}{ll}
\mathcal{A}_{4}^{\phi^{3}}[1234 \mid 1234] & \mathcal{A}_{4}^{\phi^{3}}[1234 \mid 1324] \\
\mathcal{A}_{4}^{\phi^{3}}[1234 \mid 1324] & \mathcal{A}_{4}^{\phi^{3}}[1324 \mid 1324]
\end{array}\right)^{-1}\left(\begin{array}{c}
\mathcal{A}_{4}^{B}[1234] \\
\mathcal{A}_{4}^{B}[1324]
\end{array}\right) \\
& =\frac{1}{m^{2}} \mathcal{A}_{4}^{A}[1234]\left(m^{2}+s_{12}\right)\left(\mathcal{A}_{4}^{B}[1234]\left(2 m^{2}+s_{12}\right)-\mathcal{A}_{4}^{B}[1324]\left(m^{2}+s_{13}\right)\right) \\
& +\frac{1}{m^{2}} \mathcal{A}_{4}^{A}[1324]\left(m^{2}+s_{13}\right)\left(-\mathcal{A}_{4}^{B}[1234]\left(m^{2}+s_{12}\right)+\mathcal{A}_{4}^{B}[1324]\left(2 m^{2}+s_{13}\right)\right) .
\end{aligned}
$$

Remarkably, this formula coincides exactly with the one we arrived at from the massive BCJ double-copy in (2.7).

Proceeding to 5-point, the explicit comparison of KLT and BCJ forms of the doublecopy can be repeated using the results of appendix B. We find that, again, the KLT kernel from the massive BCJ double-copy is precisely the inverse of massive bi-adjoint scalar amplitudes.

Generalizing this result to $n$-particle scattering, the KLT formulation of the massive double-copy takes the form

$$
\mathcal{A}_{n}^{A \otimes B}(1,2, \cdots, n)=\sum_{\alpha, \beta} \mathcal{A}_{n}^{A}[\alpha]\left(\mathcal{A}_{n}^{\phi^{3}}\right)^{-1}[\alpha \mid \beta] \mathcal{A}_{n}^{B}[\beta]
$$

where $\alpha$ and $\beta$ now range over all $(n-2)$ ! DDM color orderings and $\mathcal{A}_{n}^{\phi^{3}}[\alpha \mid \beta]$ is a matrix of amplitudes of massive bi-adjoint scalar theory.

We will now close this subsection with a brief discussion about the relation between the massless KLT formula (2.11) and the $m \rightarrow 0$ limit of the new massive KLT formula (2.16). 
Before doing so there is a subtlety in this discussion we should address. For generic massive field theories, in particular those containing particles with spin $\geq 1$, the naive massless limit, with $m \rightarrow 0$ and all couplings held fixed, may not exist. As reviewed in detail in appendix A, for any model, a regular massless limit may be defined as an appropriate double-scaling or decoupling limit. Throughout this paper, this is simply referred to as the massless limit.

Expanding the kernel of the $n=4$ formula around the $m=0$ limit gives

$$
\begin{aligned}
\mathcal{A}_{4}^{A \otimes B}(1,2,3,4)= & \frac{1}{m^{2}}\left(s_{12} \mathcal{A}_{4}^{A}[1234]-s_{13} \mathcal{A}_{4}^{A}[1324]\right)\left(s_{12} \mathcal{A}_{4}^{B}[1234]-s_{13} \mathcal{A}_{4}^{B}[1324]\right) \\
& +\left(3 s_{12} \mathcal{A}_{4}^{A}[1234] \mathcal{A}_{4}^{B}[1234]+3 s_{13} \mathcal{A}_{4}^{A}[1324] \mathcal{A}_{4}^{B}[1324]\right. \\
& \left.+s_{14}\left(\mathcal{A}_{4}^{A}[1234] \mathcal{A}_{4}^{B}[1324]+\mathcal{A}_{4}^{A}[1324] \mathcal{A}_{4}^{B}[1234]\right)\right)+\mathcal{O}\left(m^{2}\right)
\end{aligned}
$$

The coefficient of the leading $\mathcal{O}\left(\mathrm{m}^{-2}\right)$ term is recognizable as a product of factors that would vanish if the models $A$ and $B$ were massless and satisfied the fundamental BCJ relations. If we take the massless limit of models $A$ and $B$, which is finite as $m \rightarrow 0$ by assumption, then this term in the KLT formula is divergent. There are then two logical possibilities: $(i)$ this leading term is non-zero, and so the double-scalings needed to regularize the massless limit before and after the double-copy do not agree, or $(i i)$ this term is zero because at least one of the models $A$ or $B$ satisfy the fundamental BCJ identity in the massless limit. In the latter case, if both $A$ and $B$ satisfy the fundamental BCJ identity in the massless limit, then the massive KLT formula reduces to the familiar massless KLT relation,

$$
\mathcal{A}_{4}^{A \otimes B}(1,2,3,4)=-s_{14} \mathcal{A}^{A}[1234] \mathcal{A}^{B}[1324]+\mathcal{O}\left(m^{2}\right),
$$

and we see that the double-copy and the massless limit commute. Since this required an additional assumption, we conclude that this property does not follow from color-kinematics duality alone.

\subsection{Spurious singularities}

We have seen so far that our proposed massive KLT formula (2.16) does not require BCJtype constraints in order to define a double-copy. In appendix $\mathrm{C}$ we prove that, assuming models $A$ and $B$ have the usual locality and factorization properties, the formula (2.16) contains only simple poles at the locations of physical singularities and the resulting doublecopy amplitudes factor correctly into the product of lower point amplitudes. These properties might suggest that (2.16) can double-copy any massive theory into a different local theory, but this is not the case. Locality requires not only that the amplitude should contain physical singularities, but also that there are no additional spurious singularities. Since these do not occur in the partial amplitudes of models $A$ and $B$ by assumption, they can only appear in the KLT kernel, which we will now analyze in detail.

In general the inverse of a matrix, $M^{-1}$, equals the matrix of cofactors times $1 / \operatorname{det} M$, where the cofactors are sums of products of elements of $M$. In the massive (massless) KLT kernel, $M=\mathcal{A}_{n}^{\phi^{3}}$, and the elements are physical scattering amplitudes of the massive (massless) bi-adjoint scalar theory, which have only physical singularities. Thus, any spurious singularities in the kernel must be a result of zeros of det $\mathcal{A}_{n}^{\phi^{3}}$. 
Let us first understand how such potential spurious singularities are avoided in the massless KLT kernel. Here the BCJ relations restrict us to a subset of the DDM basis, and as a result, not all physical poles are present in $\mathcal{A}_{n}^{\phi^{3}}[\alpha \mid \beta]$. Thus some physical poles must appear as zeros of the determinant of $\mathcal{A}_{n}^{\phi^{3}}[\alpha \mid \beta]$, while others will appear in the matrix of cofactors. For example at 4-point we have

$$
\begin{aligned}
\mathcal{A}_{4}^{\phi^{3}}[1234 \mid 1234] & =-\frac{s_{13}}{s_{12} s_{14}} \\
\Rightarrow \operatorname{det} \mathcal{A}_{4}^{\phi^{3}}[1234 \mid 1234] & =-\frac{s_{13}}{s_{12} s_{14}} .
\end{aligned}
$$

Thus there is one zero of the determinant $s_{13}=0$ and it is a physical pole. Due to the color-ordering constraints, consistency with locality requires that $\mathcal{A}_{4}[1234]$ does not have a pole at $s_{13}=0$. The missing pole in the double-copied amplitude is therefore provided by the zero of the determinant at $s_{13}=0$.

A similar structure exists at 5-point. Consider BCJ orderings like that in [7], [13524] and [13542]. This gives

$$
\operatorname{det} \mathcal{A}_{5}^{\phi^{3}}[\alpha \mid \beta]=-\frac{s_{23} s_{15} s_{34}}{s_{12} s_{13} s_{14} s_{24} s_{45} s_{35} s_{25}} .
$$

Again we find that zeros of the determinant $s_{23}=s_{15}=s_{34}=0$, all correspond to physical poles. In addition, the color-ordering requires $\mathcal{A}_{5}[13524]$ and $\mathcal{A}_{5}[13542]$ to have no poles at these locations. Thus, also at 5-point, the zeros of the determinant contribute simple physical poles at locations otherwise excluded by color-ordering constraints.

Let us now investigate what happens to our proposed massive KLT formula at 4-point. We begin by choosing a DDM basis of orderings ([1234], [1324]). This gives,

$$
\operatorname{det} \mathcal{A}_{4}^{\phi^{3}}[\alpha \mid \beta]=\frac{m^{2}}{\left(s_{12}+m^{2}\right)\left(s_{13}+m^{2}\right)\left(s_{14}+m^{2}\right)},
$$

which has no zeros and thus no spurious pole can arise from the 4-point double-copy. The reciprocal of this determinant does contain a $1 / \mathrm{m}^{2}$ factor in the double-copied amplitude. This is exactly the factor we found at the end of the previous subsection in the expansion around the $m \rightarrow 0$ limit (2.17), and is responsible for the failure of the double-copy and massless decoupling limit to commute.

The absence of any additional kinematic zeroes in the determinant has the interesting consequence that any massive theory, satisfying the assumptions enumerated in the Introduction, can be inserted into the massive KLT formula to obtain a 4-point amplitude of a local theory.

At 5-point, we are less lucky. Consider a basis of DDM orderings [13 $\sigma(245)]$ where $\sigma$ runs over all 6 permutations of $(2,4,5)$, also used in [7]. Here we find

$$
\operatorname{det} \mathcal{A}_{5}^{\phi^{3}}[\alpha \mid \beta]=\frac{m^{8}}{\prod_{i} \mathcal{D}_{i}} \mathcal{P}\left(s_{i j}, m^{2}\right)
$$

where

$$
\begin{aligned}
\prod_{i} \mathcal{D}_{i}= & \left(m^{2}+s_{12}\right)^{2}\left(m^{2}+s_{13}\right)^{2}\left(m^{2}+s_{14}\right)^{2}\left(m^{2}+s_{23}\right)^{2}\left(m^{2}+s_{24}\right)^{2} \\
& \left(m^{2}+s_{15}\right)^{2}\left(m^{2}+s_{45}\right)^{2}\left(m^{2}+s_{35}\right)^{2}\left(m^{2}+s_{25}\right)^{2}\left(m^{2}+s_{34}\right)^{2},
\end{aligned}
$$


and

$$
\begin{aligned}
\mathcal{P}\left(s_{i j}, m^{2}\right)= & 320 m^{8}+ \\
+ & 36 m^{6}\left(9 s_{12}+4\left(s_{13}+s_{14}+s_{23}+s_{24}\right)\right) \\
+m^{4}( & 117 s_{12}^{2}+108 s_{12}\left(s_{13}+s_{14}+s_{23}+s_{24}\right)+4\left(s_{13}\left(13 s_{14}+4 s_{23}+17 s_{24}\right)\right. \\
& \left.\left.+4 s_{13}^{2}+4 s_{14}^{2}+17 s_{14} s_{23}+4 s_{14} s_{24}+4 s_{23}^{2}+13 s_{23} s_{24}+4 s_{24}^{2}\right)\right) \\
+ & m^{2}\left(9 s_{12}^{3}+13 s_{12}^{2}\left(s_{13}+s_{14}+s_{23}+s_{24}\right)+s_{12}\left(s_{13}\left(10 s_{14}+6 s_{23}+17 s_{24}\right)\right.\right. \\
& \left.+4 s_{13}^{2}+4 s_{14}^{2}+s_{14}\left(17 s_{23}+6 s_{24}\right)+2\left(2 s_{23}+s_{24}\right)\left(s_{23}+2 s_{24}\right)\right) \\
& +2\left(s_{13}^{2}\left(s_{14}+2 s_{24}\right)+s_{13}\left(s_{14}^{2}+s_{14}\left(s_{23}+s_{24}\right)+s_{24}\left(s_{23}+2 s_{24}\right)\right)\right. \\
& \left.\left.+s_{23}\left(s_{24}\left(s_{14}+s_{23}\right)+2 s_{14}\left(s_{14}+s_{23}\right)+s_{24}^{2}\right)\right)\right) \\
+ & 2 s_{24}\left(s_{23}\left(s_{12}^{2}+s_{12}\left(s_{13}+s_{14}\right)-s_{13} s_{14}\right)+s_{12}\left(s_{12}+s_{13}\right)\left(s_{12}+s_{13}+s_{14}\right)\right) \\
+ & +\left(s_{12}\left(s_{12}+s_{13}+s_{14}\right)+s_{23}\left(s_{12}+s_{14}\right)\right)^{2}+s_{24}^{2}\left(s_{12}+s_{13}\right)^{2} .
\end{aligned}
$$

Here, $\mathcal{D}_{i}$ contains all the physical poles and $\mathcal{P}$ is a quartic polynomial in Mandelstams. Allowing one of the five independent Mandelstam variables to vary, holding the other four fixed, we find that there are four zeros of the determinant that do not correspond to physical poles. As a result, unless the amplitudes $\mathcal{A}_{5}[13 \sigma(245)]$ conspire to cancel these spurious poles when we sum over the whole DDM basis, the proposed massive KLT formula will not give us amplitudes of a local theory. We expect that the presence of spurious poles will persist at higher-point.

It is interesting to note that quartic polynomial $\mathcal{P}$ vanishes when the external momenta $p_{i}$ are restricted to three dimensions, pointing to possible relations between amplitudes in the DDM basis. Thus one cannot immediately conclude that spurious singularities arise when double-copying three-dimensional massive theories via the construction in this section.

This analysis of the equivalent KLT form of the proposed massive double-copy reveals a dangerous tension with locality. As we have argued, color-kinematics duality satisfying BCJ numerators exist (at least up to $n=5$ ) for generic models with uniform non-zero mass spectra. But such a double-copy will contain spurious singularities unless magical cancellations take place to remove them. Such cancellations will necessarily require additional relations among the DDM basis of partial amplitudes. Since there is no analogue of the usual BCJ relations, themselves a consequence of color-kinematics duality in massless models, these relations must be genuinely new constraints.

\section{Massive gravity and (Massive Yang-Mills) ${ }^{2}$}

To definitively establish that color-kinematics duality is not a sufficient condition for a double-copy to be physical, it is enough to construct a single explicit counterexample. In this section we analyze in detail the massive Yang-Mills EFT and demonstrate that a BCJ representation of the scattering amplitudes with color-kinematics duality satisfying numerators exists, at least up to 5-point. We see that 3- and 4-point scattering amplitudes 
generated by the double-copy can be interpreted as coming from a theory of dRGT massive gravity and show that at 5-point the would-be double-copied amplitude contains spurious singularities.

\subsection{Physical motivation}

To understand the model we consider and the independent physical arguments that suggest a massive double-copy should be sensible, it is useful to begin with a slightly more general class of models. We consider models with a global $U(N)$ symmetry with a spectrum of spin-1 states of mass $m$ transforming in the adjoint representation. To ensure the existence of a standard BCJ representation (1.11), we will restrict to interactions in which the color indices are contracted using only the (totally anti-symmetric) structure constants $f^{a b c}$. The most general such model with parity-conserving interaction terms of mass dimension up to four is given by the Lagrangian ${ }^{5}$

$$
\mathcal{L}=-\frac{1}{4}\left(\partial_{[\mu} A_{\nu]}^{a}\right)^{2}-\frac{1}{2} m^{2} A_{\mu}^{a} A^{a \mu}-g f^{a b c} A_{\mu}^{a} A_{\nu}^{b} \partial^{\mu} A^{c \nu}-\frac{1}{4} g^{\prime} f^{a b e} f^{c d e} A_{\mu}^{a} A^{\mu c} A_{\nu}^{b} A^{\nu d} .
$$

Models of this kind with massive spinning states are generically only valid as low-energy effective descriptions. The associated scattering amplitudes violate perturbative unitarity bounds at a parametrically low energy scale unless special tunings of couplings are made or additional states such as Higgs bosons are introduced to soften the UV behaviour. An efficient way to observe this is to study high-energy fixed angle, 2-to-2 scattering amplitudes. Here we use explicit center-of-mass frame kinematics with polarization vectors,

$$
\begin{aligned}
\epsilon_{\mu}^{( \pm)}\left(p^{i}\right) & =\left(0, \mp \cos \theta^{i},-i, \pm \sin \theta^{i}\right) \\
\epsilon_{\mu}^{(0)}\left(p^{i}\right) & =\frac{1}{m}\left(p, E \sin \theta^{i}, 0, E \cos \theta^{i}\right),
\end{aligned}
$$

and momenta

$$
p_{\mu}^{i}=\left(E, p \sin \theta^{i}, 0, p \cos \theta^{i}\right),
$$

with $i=1,2,3$, 4 labeling the external particles scattering at angles $\theta^{1}=0, \theta^{2}=\pi, \theta^{3}=\theta$, $\theta^{4}=\theta-\pi$. The worst behaved choice for the polarizations is given by purely longitudinal scattering ${ }^{6}$

$$
\begin{aligned}
\mathcal{A}(0000)= & \frac{1}{4 m^{4}}\left(g^{2}-g^{\prime}\right)\left[c_{12}\left(2 s^{2}+2 s t-t^{2}\right)+c_{13}\left(s^{2}-2 s t-2 t^{2}\right)\right] \\
& +\frac{1}{4 m^{2}}\left[c_{12}\left(4 g^{\prime}(2 s+3 t)-g^{2}(8 s+13 t)\right)+c_{13} s\left(4 g^{\prime}-3 g^{2}\right)\right]+\mathcal{O}\left(s^{0}\right)
\end{aligned}
$$

where we have parametrized the expression in terms of the $m \rightarrow 0$ limit of the Mandelstam invariants

$$
s \equiv 4 E^{2}, \quad t \equiv 2 E^{2}(\cos (\theta)-1) .
$$

\footnotetext{
${ }^{5}$ In this paper we will use the Lie algebra conventions $\left[T^{a}, T^{b}\right]=i f^{a b c} T^{c}$ and $\operatorname{Tr}\left[T^{a} T^{b}\right]=\delta^{a b}$.

${ }^{6}$ Here we are using a shorthand notation $\mathcal{A}\left(s_{1} s_{2} s_{3} s_{4}\right) \equiv \mathcal{A}_{4}\left(1_{s_{1}}^{a_{1}}, 2_{s_{2}}^{a_{2}} \rightarrow 3_{s_{3}}^{a_{3}}, 4_{s_{4}}^{a_{4}}\right)$, where $a_{i}$ are adjoint indices and $s_{i}=+,-, 0$ is the polarization.
} 
We see that for generic values of $g^{\prime}$ the scattering amplitudes grow like $E^{4}$ at high-energies, but for a specific tuning, $g^{\prime}=g^{2}$, this is improved to $E^{2}$. If this tuning is made, the generic Lagrangian (3.1) simplifies to

$$
\mathcal{L}=-\frac{1}{4}\left(F_{\mu \nu}^{a}\right)^{2}-\frac{1}{2} m^{2} A_{\mu}^{a} A^{a \mu},
$$

where

$$
F_{\mu \nu}^{a} \equiv \partial_{[\mu} A_{\nu]}^{a}+g f^{a b c} A_{\mu}^{b} A_{\nu}^{c},
$$

and defines the model we will study in this section under the name massive Yang-Mills.

The improved high-energy behaviour of this tuning has a nice physical explanation. The massive Yang-Mills model has a simple (perturbative) UV completion as a particular limit of a Higgsed gauge theory. We begin with a model of scalar fields $\phi^{a a^{\prime}}$ transforming in the bi-adjoint representation of $U(N)_{L} \times U(N)_{R}$ with a Higgs potential

$$
\mathcal{L}=-\frac{1}{2}\left(\partial_{\mu} \phi^{a a^{\prime}}\right)^{2}+\lambda v^{2} \phi^{a a^{\prime}} \phi^{a a^{\prime}}-\frac{\lambda}{2}\left(\phi^{a a^{\prime}} \phi^{a a^{\prime}}\right)^{2} .
$$

When $\lambda>0$ and $v^{2}>0$, the $U(N)_{L} \times U(N)_{R}$ symmetry is spontaneously broken to a $U(N)$ subgroup. Without loss of generality the vacuum expectation value can be taken to have the form

$$
\left\langle\phi^{a a^{\prime}}\right\rangle=\frac{v}{N} \delta^{a a^{\prime}},
$$

for which the unbroken subgroup $U(N)_{V}$ is generated by the "vector-like" combinations ${ }^{7}$

$$
\left(T_{V}^{i}\right)^{a a^{\prime} b b^{\prime}}=\left(T_{L}^{i}\right)^{a b} \delta^{a^{\prime} b^{\prime}}+\delta^{a b}\left(T_{R}^{i}\right)^{a^{\prime} b^{\prime}} .
$$

If we gauge the orthogonal, broken "axial-like" subgroup $U(N)_{A}$ generated by

$$
\left(T_{A}^{i}\right)^{a a^{\prime} b b^{\prime}}=\left(T_{L}^{i}\right)^{a b} \delta^{a^{\prime} b^{\prime}}-\delta^{a b}\left(T_{R}^{i}\right)^{a^{\prime} b^{\prime}},
$$

then in unitary gauge the associated $U(N)_{A}$ gauge bosons acquire masses $m_{A} \sim g v$, while preserving the unbroken global $U(N)_{V}$ symmetry under which they transform in the adjoint representation. The remaining $N^{2}\left(N^{2}-1\right)$ Higgs scalars have masses $m_{H} \sim \lambda^{1 / 2} v$, and in the limit $\lambda \rightarrow \infty$ with $v$ held fixed, decouple, with the low-energy dynamics of the massive vector bosons described by the massive Yang-Mills EFT.

The Goldstone boson equivalence theorem [28] tells us that the high-energy scattering of longitudinal vector modes of a spontaneously broken gauge theory must match the highenergy limit of a coset sigma model describing the same symmetry breaking pattern. In this case the coset is $\left(U(N)_{L} \times U(N)_{R}\right) / U(N)_{V}$, which is coincidentally the coset defining Chiral Perturbation Theory $(\chi \mathrm{PT})[29]$, with the well-known Lagrangian

$$
\mathcal{L}=\frac{f_{\pi}^{2}}{2} \operatorname{Tr}\left[\partial_{\mu} U^{\dagger} \partial^{\mu} U\right], \quad U(x) \equiv \exp \left(\frac{i}{f_{\pi}} T^{a} \pi^{a}(x)\right) .
$$

\footnotetext{
${ }^{7}$ Here the adjoint generators are defined as $\left(T_{L}^{i}\right)^{a b}=f^{i a b}$ and $\left(T_{R}^{i}\right)^{a^{\prime} b^{\prime}}=f^{i a^{\prime} b^{\prime}}$.
} 
The 2-to-2 scattering amplitude in this model is given by the simple expression

$$
\mathcal{A}_{4}(1,2,3,4)=\frac{1}{4 f_{\pi}^{2}}\left(-c_{12} t+c_{13} s\right),
$$

which precisely matches (3.4) in the limit $g^{\prime}=g^{2}$, if the pion decay constant is identified as $f_{\pi} \sim m / g$.

Massive Yang-Mills is not only a special EFT because it has softer than expected highenergy growth. As the above discussion indicates, in the high-energy limit the scattering amplitudes coincide with those of $\chi \mathrm{PT}$, which is among the special class of massless models exhibiting color-kinematics duality [4, 27], as can be verified explicitly using (3.13). As a consequence, in the high-energy limit the massive Yang-Mills amplitudes can be doublecopied to give the scattering amplitudes of the special Galileon [30],

$$
\left(\lim _{E \gg m} \mathcal{A}_{n}^{\mathrm{mYM}}\right) \otimes\left(\lim _{E \gg m} \mathcal{A}_{n}^{\mathrm{mYM}}\right)=\mathcal{A}_{n}^{\mathrm{sGal}} .
$$

Galileons were originally discovered in the context of the DGP model of modified gravity [31], but were later found to arise naturally in the decoupling limit of ghost-free massive gravity [32]. On the basis of this observation, it seems natural to speculate that there exists some model of a massive spin- 2 or massive gravity, which matches the special Galileon amplitudes at high-energies and can be constructed as a double-copy

$$
\mathcal{M}_{n}^{\mathrm{mGrav}} \equiv \mathcal{A}_{n}^{\mathrm{mYM}} \otimes_{m} \mathcal{A}_{n}^{\mathrm{mYM}}
$$

An immediate problem with this is that we do not know what the symbol $\otimes_{m}$, denoting a massive double-copy, is supposed to mean. One property it should have, if this story is self-consistent, is that it commutes with the high-energy limit, meaning

$$
\lim _{E \gg m}\left(\mathcal{A}_{n}^{\mathrm{mYM}} \otimes_{m} \mathcal{A}_{n}^{\mathrm{mYM}}\right) \stackrel{!}{=}\left(\lim _{E \gg m} \mathcal{A}_{n}^{\mathrm{mYM}}\right) \otimes\left(\lim _{E \gg m} \mathcal{A}_{n}^{\mathrm{mYM}}\right)
$$

where $\otimes$ on the right-hand-side is the familiar massless double-copy. In the Introduction (1.11), we described a natural generalization of the BCJ double-copy based on colorkinematics duality, to models with massive states, and in section 2 constructed an equivalent KLT-like formula. In this section we will demonstrate explicitly that such a doublecopy does not have the property (3.16) and moreover, for $n>4$ does not produce a physical scattering amplitude that can be matched to a local Lagrangian.

\subsection{3-point amplitudes and asymptotic states}

Before considering the dynamical content of the double-copy, we first need to understand the mapping of states in the asymptotic Hilbert space. Massive Yang-Mills is a model of a massive vector boson, with 3 on-shell degrees of freedom in $d=4$. The Hilbert space of asymptotic one-particle states is spanned by the space of plane-wave solutions to the linearized equations of motion. In the present context it is convenient to represent the basis of linearly independent plane-wave solutions using the massive spinor formalism of [33]. In 
this approach, the 3 independent spin states are collected together into a rank-2, totally symmetric $S U(2)$ little group tensor. Explicitly,

$$
A_{\mu}^{a I J}(x)=c^{a} \epsilon_{\mu}^{I J}(p) e^{i p \cdot x}, \quad \text { where } \quad \epsilon_{\mu}^{I J}(p)=-\frac{1}{2 \sqrt{2}} \tilde{\lambda}_{\dot{\alpha}}^{(I} \bar{\sigma}_{\mu}^{\dot{\alpha} \alpha} \lambda_{\alpha}^{J)} .
$$

The double-copy of such a plane-wave solution is given simply by replacing the color factor $c^{a}$ with a second copy of the polarization vector,

$$
A_{\mu}^{a I J}(x) \otimes A_{\nu}^{b K L}(x)=\mathfrak{h}_{\mu \nu}^{I J K L}(x) \equiv \epsilon_{\mu}^{I J}(p) \epsilon_{\nu}^{K L}(p) e^{i p \cdot x} .
$$

Where (3.17) transforms in an irreducible representation of $S U(2)$, the double-copy (3.18) transforms in a reducible representation. Such a plane-wave double-copy is equivalent to a tensor product of one-particle Hilbert spaces, for which standard decomposition of representations of $S U(2)$ gives the physical spectrum of the double-copy

$$
\mathbf{3} \otimes \mathbf{3}=\mathbf{5} \oplus \mathbf{3} \oplus \mathbf{1} .
$$

Hence we expect the double-copy of massive Yang-Mills to describe a model of a massive graviton $h_{\mu \nu}$ (spin-2) coupled to a massive Kalb-Ramond two-form $B_{\mu \nu}$ (spin-1) and a massive dilaton $\phi$ (spin-0). It is most convenient to first calculate the scattering amplitudes for the reducible $\mathfrak{h}$-states, and project out the physical states as needed. To extract the physical spectrum of the double-copy we use the following projection operators ${ }^{8}$

$$
\begin{aligned}
\left(P_{h}\right)_{I_{1} I_{2} J_{1} J_{2}}^{K_{1} K_{2} K_{3} K_{4}} & =\frac{1}{24} \delta_{I_{1} I_{2} J_{1} J_{2}}^{\left(K_{1} K_{2} K_{3} K_{4}\right)}, \quad\left(P_{B}\right)_{I_{1} I_{2} J_{1} J_{2}}^{K_{1} K_{2}}=\frac{1}{\sqrt{2}} \epsilon_{I_{1} J_{1}} \delta_{I_{2} J_{2}}^{\left(K_{1} K_{2}\right)}, \\
\left(P_{\phi}\right)_{I_{1} I_{2} J_{1} J_{2}} & =\frac{1}{\sqrt{3}} \epsilon_{I_{1} J_{1}} \epsilon_{I_{2} J_{2}} .
\end{aligned}
$$

The physical polarization tensor of the two-form is antisymmetric $\epsilon_{\mu \nu}^{(B)}=-\epsilon_{\nu \mu}^{(B)}$, and consequently gives a non-vanishing contribution to amplitudes in the double-copy only if there are an even number of such states. Equivalently, the two-form has a $\mathbb{Z}_{2}$ symmetry, which allows us to form a consistent truncation containing only the graviton and dilaton modes.

Since the polarization tensors in the truncated model are symmetric we can represent the amplitudes using a convenient shorthand. We suppress the little-group indices by making the replacement $\epsilon_{\mu}^{I_{i} I_{i}}\left(p_{i}\right) \rightarrow z_{\mu}^{i}$; the amplitude is then a rational function of the following elementary building blocks:

$$
p_{i j} \equiv p_{\mu}^{i} p^{j \mu}, \quad z_{i j} \equiv z_{\mu}^{i} z^{j \mu}, \quad z p_{i j} \equiv z_{\mu}^{i} p^{j \mu} .
$$

Extracting the physical graviton and dilaton states amounts to the replacement rules,

$$
\begin{aligned}
& z_{\mu}^{i} z_{\nu}^{i} \rightarrow \epsilon_{\mu \nu}\left(p_{i}\right) \quad \text { (Massive Graviton) } \\
& z_{\mu}^{i} z_{\nu}^{i} \rightarrow \frac{1}{\sqrt{3}}\left(\eta_{\mu \nu}+\frac{p_{i \mu} p_{i \nu}}{m^{2}}\right) \quad \text { (Massive Dilaton). }
\end{aligned}
$$

\footnotetext{
${ }^{8}$ The normalization constants can be fixed by requiring that the completeness relation for polarizations gives the same sum over states before and after projecting onto physical states.
} 
We begin with the double-copy of 3-point scattering amplitudes. This is of course unconstrained by color-kinematics duality, but will be important for reconstructing the massive gravity Lagrangian from the 4-point amplitudes. A local BCJ representation of the massive Yang-Mills amplitudes can be efficiently constructed using the Feynman rules given in appendix D. The cubic Yang-Mills amplitude is given by

$$
\mathcal{A}_{3}=2 g\left(z_{23} z p_{12}+z_{13} z p_{23}+z_{12} z p_{31}\right) \text {. }
$$

The gravitational amplitude is given by squaring the Yang-Mills amplitude and replacing the coupling constants as $g^{2} \rightarrow \frac{1}{2 M_{p}}$, giving

$$
\mathcal{M}_{3}=\frac{2}{M_{p}}\left(z_{23} z p_{12}+z_{13} z p_{23}+z_{12} z p_{31}\right)^{2} .
$$

Using (3.22) we can extract from this the cubic amplitudes for physical states. The on-shell cubic amplitude for 3 gravitons is formally identical to the massless case, given by:

$$
\begin{aligned}
\mathcal{M}\left(1_{h}, 2_{h}, 3_{h}\right)=\frac{2}{M_{p}}( & \epsilon_{1 \mu \nu} \epsilon_{2}{ }^{\mu \nu} \epsilon_{3 \alpha \beta} p_{1}{ }^{\alpha} p_{1}{ }^{\beta}+2 p_{2}{ }^{\mu} \epsilon_{1 \mu \nu} \epsilon_{2}{ }^{\nu \alpha} \epsilon_{3 \alpha \beta} p_{1}{ }^{\beta} \\
& + \text { cyclic permutations of }(1,2,3)) .
\end{aligned}
$$

The amplitude for 2 gravitons and 1 dilaton is given by

$$
\mathcal{M}_{3}\left(1_{h}, 2_{h}, 3_{\phi}\right)=-\frac{\sqrt{3}}{2 M_{p}} m^{2} \epsilon_{1 \mu \nu} \epsilon_{2}{ }^{\mu \nu} .
$$

We see that this expression vanishes as $m \rightarrow 0$, recovering the expected massless amplitude. It is interesting to note that the $\mathbb{Z}_{2}$ dilaton parity of the massless double-copy only emerges in the massless limit. Therefore when $m \neq 0$ we cannot make a further consistent truncation to the gravity sector. The on-shell cubic amplitudes for 1 graviton and 2 dilatons is given by

$$
\mathcal{M}_{3}\left(1_{h}, 2_{\phi}, 3_{\phi}\right)=\frac{3}{2 M_{p}} \epsilon_{1 \mu \nu} p_{2}{ }^{\mu} p_{2}{ }^{\nu} .
$$

This vertex appears in both the massive and massless cases. The on-shell cubic amplitude for 3 dilatons is given by

$$
\mathcal{M}_{3}\left(1_{\phi}, 2_{\phi}, 3_{\phi}\right)=-\frac{11 \sqrt{3}}{8 M_{p}} m^{2} .
$$

This cubic dilaton vertex is also unique to the massive case and does not appear in the massless case.

\subsection{4-point amplitudes and high energy behavior}

A BCJ representation of the 4-point amplitude is straightforwardly generated from the Feynman rules in appendix D. This gives the following massive kinematic numerators

$$
\begin{aligned}
n_{12}= & {\left[\left(\epsilon_{1} \cdot \epsilon_{2}\right) p_{1}^{\mu}+2\left(\epsilon_{1} \cdot p_{2}\right) \epsilon_{2}^{\mu}-(1 \leftrightarrow 2)\right]\left(g_{\mu \nu}+\frac{\left(-p_{1 \mu}-p_{2 \mu}\right)\left(p_{3 \nu}+p_{4 \nu}\right)}{m^{2}}\right) } \\
& \times\left[\left(\epsilon_{3} \cdot \epsilon_{4}\right) p_{3}{ }^{\nu}+2\left(\epsilon_{3} \cdot p_{4}\right) \epsilon_{4}^{\nu}-(3 \leftrightarrow 4)\right] \\
& +\left(s+m^{2}\right)\left[\left(\epsilon_{1} \cdot \epsilon_{3}\right)\left(\epsilon_{2} \cdot \epsilon_{4}\right)-\left(\epsilon_{1} \cdot \epsilon_{4}\right)\left(\epsilon_{2} \cdot \epsilon_{3}\right)\right],
\end{aligned}
$$


with the first two lines coming from the exchange diagrams and the third line coming from the contact diagram. The other numerators are found by taking

$$
n_{13}=\left.n_{12}\right|_{1 \rightarrow 3 \rightarrow 2 \rightarrow 1}, \quad n_{14}=\left.n_{12}\right|_{1 \rightarrow 2 \rightarrow 3 \rightarrow 1} .
$$

The $1 / m^{2}$ term in the massive vector propagator vanishes, and so these numerators are formally identical to the Feynman rule-generated expressions for massless Yang-Mills. As a consequence of this formal equivalence, together with the fact that at 4-point in massless Yang-Mills, all generalized gauges satisfy the kinematic Jacobi identity, we find that this Feynman rule generated expression for the mass deformed numerators (3.29) and (3.30), just happens to be in the unique generalized gauge to satisfy the massive kinematic Jacobi relation

$$
n_{12}+n_{13}+n_{14}=0
$$

The 4-point massive gravity amplitude is then given by

$$
\mathcal{M}_{4}=\frac{1}{4 M_{p}^{2}}\left(\frac{n_{12}^{2}}{s_{12}+m^{2}}+\frac{n_{13}^{2}}{s_{13}+m^{2}}+\frac{n_{14}^{2}}{s_{14}+m^{2}}\right) .
$$

The explicit expressions for the physical scattering amplitudes are rather complicated and are given explicitly in appendix E. ${ }^{9}$

We expect the double-copy procedure for massive Yang-Mills to give a ghost-free theory of massive gravity. ${ }^{10}$ Generic ghost free massive gravity without coupling to a dilaton, also known as dRGT, propagates 5 degrees of freedom, has two free parameters in $D=4$, and is given by the action

$$
S=\frac{M_{P}^{D-2}}{2} \int d^{D} x\left[(\sqrt{-g} R)-\sqrt{-g} \frac{1}{4} m^{2} W(g, \mathcal{K})\right],
$$

where

$$
W(g, \mathcal{K})=\sum_{n=2}^{n=D} \alpha_{n} \mathcal{L}_{n}^{T D}(\mathcal{K}),
$$

brackets mean trace with respect to the full metric, $\alpha_{2}=-4$, and the rest of the coefficients are arbitrary $[23,35]$. The tensor $\mathcal{K}_{\nu}^{\mu}(g, H)$ is given by

$$
\mathcal{K}_{\nu}^{\mu}=\delta_{\nu}^{\mu}-\sqrt{\delta_{\nu}^{\mu}-H_{\nu}^{\mu}}=\sum_{n=1}^{\infty} d_{n}\left(H^{n}\right)_{\nu}^{\mu}, \quad d_{n}=-\frac{(2 n) !}{(1-2 n)(n !)^{2} 4^{n}},
$$

where indices are raised by the full metric $g_{\nu}^{\mu}=\gamma_{\nu}^{\mu}+h_{\nu}^{\mu}$, the background metric is $\gamma_{\nu}^{\mu}$, and $H_{\nu}^{\mu}=g_{\nu}^{\mu}-\tilde{\gamma}_{\nu}^{\mu}$ is the Stückelberg replacement for $h_{\nu}^{\mu}$. The quantity $\mathcal{L}_{n}^{T D}(\Pi)$ can be written as total derivatives when $\Pi=\partial_{\mu} \partial_{\nu} \phi$. These total derivative combinations are unique up to an overall constant and can be found using the recursion relation

$$
\mathcal{L}_{n}^{T D}(\Pi)=-\sum_{m=1}^{n}(-1)^{m} \frac{n !}{(n-m) !} \Pi_{\mu \nu}^{m} \mathcal{L}_{n-m}^{T D}(\Pi),
$$

with $\mathcal{L}_{0}^{T D}=1$.

\footnotetext{
${ }^{9}$ These results are in agreement with those that appeared recently in [25].

${ }^{10}$ See [34] for a review of massive gravity.
} 
Massive gravity with the most generic potential without the dRGT tuning has an extra scalar degree of freedom that is ghostly and 4-point scattering amplitudes that grow with center-of-mass energy like $E^{10}$. However, the dRGT tuning, which leaves only 2 free parameters, removes the ghostly degree of freedom and improves the high energy behavior to scale with energy as $E^{6}[36,37]$. Another common parameterization of dRGT massive gravity is given in [38]. The leading high energy behavior in this parameterization, for the tree-level 4-point amplitude for dRGT massive gravity, is given by:

$$
\begin{aligned}
\mathcal{M}\left(1^{+} 1^{+} 1^{+} 1^{+}\right) & =-\frac{3}{32}\left(1-4 c_{3}\right) s^{3} \\
\mathcal{M}\left(1^{+} 1^{+} 1^{-} 1^{-}\right) & =\mathcal{M}\left(1^{-} 1^{-} 1^{+} 1^{+}\right)=\frac{9}{32}\left(1-4 c_{3}\right)^{2} s t(s+t) \\
\mathcal{M}\left(2^{+} 000\right) & =\frac{1}{\sqrt{6}}\left(c_{3}+8 d_{5}\right) s t(s+t) \\
\mathcal{M}\left(1^{+} 1^{+} 00\right) & =\frac{1}{32} s\left(2\left(1-8 c_{3}+48 c_{3}^{2}+64 d_{5}\right) t(s+t)-3\left(1-4 c_{3}\right)^{2} s^{2}\right) \\
\mathcal{M}\left(1^{+} 1^{-} 00\right) & =\frac{1}{96} s\left(\left(1+12 c_{3}\right)^{2}+384 d_{5}\right) s t(s+t) \\
\mathcal{M}(0000) & =\frac{1}{6}\left(1+4 c_{3}\left(9 c_{3}-1\right)+64 d_{5}\right) s t(s+t),
\end{aligned}
$$

where the polarization tensors, $\epsilon_{\mu \nu}^{(a)}$, have been split into two tensor modes $\left(a=2^{+}, 2^{-}\right)$, two vector modes $\left(a=1^{+}, 1^{-}\right)$, and one scalar mode $(a=0)$, the relation between the free parameters of dRGT are given by:

$$
\alpha_{3}=-2 c_{3} \text { and } \alpha_{4}=-4 d_{5},
$$

and the polarization tensors are chosen to be:

$$
\begin{aligned}
\epsilon_{\mu \nu}^{(2 \pm)} & =\epsilon_{\mu}^{( \pm)} \epsilon_{\nu}^{( \pm)} \\
\epsilon_{\mu \nu}^{(1 \pm)} & =\frac{1}{\sqrt{2}}\left(\epsilon_{\mu}^{( \pm)} \epsilon_{\nu}^{(0)}+\epsilon_{\mu}^{(0)} \epsilon_{\nu}^{( \pm)}\right) \\
\epsilon_{\mu \nu}^{(0)} & =\frac{1}{\sqrt{6}}\left(\epsilon_{\mu}^{(+)} \epsilon_{\nu}^{(-)}+\epsilon_{\mu}^{(-)} \epsilon_{\nu}^{(+)}+2 \epsilon_{\mu}^{(0)} \epsilon_{\nu}^{(0)}\right) .
\end{aligned}
$$

Indeed the 3-point amplitude (3.24) corresponds to dRGT massive gravity with

$$
\alpha_{3}=-\frac{1}{2} \text { or } \quad c_{3}=\frac{1}{4} .
$$

This value is also the one picked out in the eikonal approximation analysis needed to avoid superluminal propagation as shown in [39] and is the "partially massless" $\alpha_{3}[23,40]$.

With the new cubic vertices that appear in the massive case, there are new scattering channels that appear in the quartic amplitudes that would not appear in the massless case. In agreement with the general discussion in section $\mathrm{C}$, we find that all quartic amplitudes factorize properly on the poles into products of the corresponding 3-point amplitudes. For example, in the 4-graviton scattering amplitude, we find contributions from diagrams corresponding to the $s, t, u$ channels mediated by both a massive graviton and a dilaton, 
due to the non-vanishing cubic coupling with 2 gravitons and 1 dilaton. The 4-graviton amplitude matches that of massive gravity with the coefficients

$$
\alpha_{4}=\frac{7}{48} \text { or } d_{5}=-\frac{7}{192},
$$

plus the additional channels mediated by the dilaton.

At first glance, it may appear that a field redefinition could mix the cubic $h h \phi$ vertex and massive gravity quartic interactions, leading to the choice of $\alpha_{4}$ to not be uniquely specified. Since amplitudes are unaffected by field redefinition, we consider the difference between the double-copied amplitude and the dRGT massive gravity amplitude with $\alpha_{3}=$ $-\frac{1}{2}$ and $\alpha_{4}$ left unspecified. We find terms proportional to $\sim\left(48 \alpha_{4}-7\right) \operatorname{Tr}\left[\epsilon_{1} \cdot \epsilon_{2} \cdot \epsilon_{3} \cdot \epsilon_{4}\right]$. This structure cannot be altered by introducing scalar channel diagrams and thus, requiring that it vanish picks out the remaining parameter to be $\alpha_{4}=\frac{7}{48}$.

The leading high energy behavior of the amplitudes for graviton-graviton scattering in the massive double-copy goes as:

$$
\begin{aligned}
\mathcal{M}\left(2^{+} 000\right) & =-\frac{1}{24 \sqrt{6}} s t(s+t) \\
\mathcal{M}\left(1^{+} 1^{+} 00\right) & =-\frac{1}{48} s t(s+t) \\
\mathcal{M}\left(1^{+} 1^{-} 00\right) & =\frac{1}{48} s t(s+t) \\
\mathcal{M}(0000) & =\frac{7}{144} s t(s+t) .
\end{aligned}
$$

For the value of $c_{3}$ picked out by the double-copy, the high energy behavior of the 4point amplitudes for massive gravity, (3.37) through (3.42), is improved for amplitudes where all the polarizations of the external particles are vector modes, scaling as $E^{4}$ rather than $E^{6}$. The dilaton affects the coefficient of $\mathcal{M}(0000)$, the amplitude where all the external particles are scalar modes. Without the dilaton, this amplitude would behave as $\mathcal{M}(0000)=-\frac{1}{72} s t(s+t)$. All other amplitudes behave as they would without the dilaton and are consistent with the above $c_{3}$ and $d_{5}$ values in expressions (3.37) through (3.42).

One immediate and important result from (3.47) is that the conjectured property (3.16) does not hold for the BCJ double-copy. In the Goldstone boson equivalence limit for massive Yang-Mills, only the spin-1 longitudinal mode contributes at $E^{2}$. If (3.16) held, we would expect only the scattering of a single scalar mode to contribute at $E^{6}$ in the double-copy. From (3.47), we see explicitly that this is not the case.

In the 4-point amplitude where all the external particles are dilatons, there will be $s, t, u$ channels mediated by a massive graviton, as well as $s, t, u$ channels mediated by a dilaton, and a 4-dilaton contact term. The massless case only has the channels mediated by the massless graviton.

The 4-point amplitude with 2 gravitons and 2 dilatons exists in the massless and massive case. In the massless case, this 4-point amplitude has graviton exchange channels via the $h \phi \phi$ and $h h h$ vertices and dilaton exchange channels via two $h \phi \phi$ vertices, plus a contact term $h h \phi \phi$. In the massive case, there will be additional graviton exchange 
channels via two $h h \phi$ vertices, as well as dilaton exchange channels via the vertices $h h \phi$ and $\phi \phi \phi$.

The 4-point amplitudes with 3 gravitons and 1 dilaton or 1 graviton and 3 dilatons are unique to the massive case and involve all possible exchange diagrams with dilaton propagators, as well as graviton propagators, and with additional $h h h \phi$ and $h \phi \phi \phi$ contact terms.

The high energy behavior of all the amplitudes scales with energy like $\sim E^{6}$ or less and the amplitudes that scale like $E^{6}$ take the special galileon form $s t(s+t)$ [30]. As another example, the leading high energy behavior of $h \phi \phi \phi$ amplitudes is shown below:

$$
\begin{aligned}
\mathcal{M}\left(2^{+} \phi \phi \phi\right) & =-\frac{s t(s+t)}{96 \sqrt{3}} \\
\mathcal{M}(0 \phi \phi \phi) & =-\frac{11 s t(s+t)}{288 \sqrt{2}} .
\end{aligned}
$$

All the 4-point graviton and dilaton amplitudes resulting from the double-copy are given in appendix E.

\subsection{5-point amplitudes and non-physical singularities}

As discussed in section C, 5-point massive gravity amplitudes constructed via the massive KLT formula are guaranteed to factorize correctly into 4- and 3-point amplitudes (listed in appendix E and section 3.2 respectively). Nonetheless as we saw at 4-point, checking factorization at 5-point is a good cross-check of our more general results, in particular those of appendix $\mathrm{C}$.

We begin by choosing a DDM basis of orderings $[13 \sigma(2,4,5)]$ where $\sigma$ runs over all possible permutations. Using the Feynman rules of massive Yang-Mills, we then calculate partial amplitudes and use the inverse of bi-adjoint scalar matrix (B.3) to construct 5-point all-graviton amplitudes. The inverse of (B.3) is unwieldy so we do the following numerical tests of factorization.

One can choose an independent basis of building blocks from the set of all $\left(\epsilon_{i} \cdot \epsilon_{j}\right)$, $\left(\epsilon_{i} \cdot p_{j}\right)$ and $\left(p_{i} \cdot p_{j}\right)$. We then assign numeric values to all these kinematic structures except one, without loss of generality let's call this $\left(p_{1} \cdot p_{2}\right)$. One can then evaluate the 5-point amplitude on this set of kinematic data and check that the residue on physical pole $\left(p_{1} \cdot p_{2}\right)=-\frac{m^{2}}{2}$ is exactly what one would expect

$$
\underset{s_{12}=-m^{2}}{\operatorname{Res}} \mathcal{M}_{5}(12345) \stackrel{!}{=} \sum_{X} \mathcal{M}_{3}\left(12\left(-P_{12}\right)_{X}\right) \times \mathcal{M}_{4}\left(\left(P_{12}\right)_{\bar{X}} 345\right),
$$

where $X$ can either be a dilaton or a graviton. As expected from the general discussion in appendix $\mathrm{C}$ we find that the would-be 5-point amplitude factors as expected on physical poles.

While the correct factorization of the 5-point amplitude is promising, we saw in section 2.2 that the KLT kernel suffers from non-physical poles arising from the determinant of the matrix of bi-adjoint scalar amplitudes. These singularities (2.24) can only be removed 
if special cancellations occur between amplitudes in the theory we are double-copying and the KLT kernel.

In the context of this explicit example, we can proceed with our numerical analysis to check for example, whether all poles in $\left(p_{1} \cdot p_{2}\right)$ are physical. This can be done by evaluating the KLT formula on an incomplete set of kinematic data that leaves $\left(p_{1} \cdot p_{2}\right)$ unspecified. One can then check if all singularities in $\left(p_{1} \cdot p_{2}\right)$ are accounted for by locality. We find that this is not the case and that the resulting 5-point amplitude $\mathcal{M}_{5}$ does have spurious poles. The singularity structure takes exactly the form (2.24) which can be recast as

$$
\mathcal{P}\left(s_{i j}, m^{2}\right)=\alpha_{1} s_{12}^{4}+\alpha_{2} s_{12}^{3}+\alpha_{3} s_{12}^{2}+\alpha_{4} s_{12}+\alpha_{5},
$$

where $\alpha_{i}$ are functions of the mass and other Mandelstam variables. Since this polynomial does not easily factor into rational roots, it is useful to choose special kinematic configurations where it factors more readily. In these cases, the exact locations of the spurious poles can be found and the amplitude evaluated on such a non-physical pole gives a nonzero residue.

Thus, no miraculous cancellations occur in massive Yang-Mills to get rid of spurious singularities. In particular this means that in its current form, massive Yang-Mills does not sensibly double-copy to massive gravity.

Furthermore, if we attempt to save the double-copy, by for example, adding a 5-point contact contribution to cancel these non-physical poles, we find no improvement. Consider for example adding a new operator at 5-point, such that

$$
\tilde{\mathcal{A}}_{5}[13542]=\mathcal{A}_{5}[13245]+\frac{\alpha g^{3}}{m^{2}}\left(p_{1} \cdot \epsilon_{3}\right)\left(\epsilon_{1} \cdot \epsilon_{2}\right)\left(\epsilon_{3} \cdot \epsilon_{5}\right),
$$

with contributions to the other orderings determined by relabeling. Here $\alpha$ is a free coefficient. The powers of $m^{2}$ have been introduced to correct the mass dimension, this would correspond to adding a term $\sim \partial A^{5}$ to the massive Yang-Mills Lagrangian.

We find that there is no way to tune $\alpha$ to remove any of the spurious singularities. Since it is unclear whether this statement still holds for arbitrary combinations of the other 28 possible $\partial A^{5}$ structures, we cannot strictly rule out the possibility of a massive YangMills 5-point operator removing non-physical poles from the KLT product. Nonetheless, our calculation is indicative that this may not be possible.

\section{Locality and the spectral condition}

We have seen that the proposed massive KLT construction (2.16) is in serious tension with locality. In general, the inverse of the matrix of KK independent massive bi-adjoint scalar amplitudes contains spurious, non-physical singularities (2.24). For the full KLT sum to be free of these non-physical singularities, additional non-trivial constraints must be imposed. These conditions are not met in the case of massive Yang-Mills, because as we saw in section 3.4, the resulting 5-point massive gravity amplitude is not local. Thus, despite the existence of color-kinematics duality satisfying numerators for all KK satisfying models, 
the resulting would-be double copies only correspond to physical amplitudes if additional constraints are imposed.

To better understand these additional constraints, let us first look at the massless case. Here the additional constraints are the fundamental BCJ relations and color-kinematics duality satisfying numerators can only be found in theories whose amplitudes are BCJcompatible. In the language of bi-adjoint scalar theory, the double-copy formulation gives rise to physical amplitudes only if the $(n-2) ! \times(n-2) !$ matrix of bi-adjoint scalar amplitudes $\mathcal{A}^{\phi^{3}}[\alpha \mid \beta]$ has rank $(n-3)$ !, which we will refer to as minimal rank. ${ }^{11}$ In addition, only theories whose amplitudes satisfy the fundamental BCJ relations, which arise as null vectors of the singular matrix of bi-adjoint scalar amplitudes, can be double-copied.

In the massive case, a matrix of bi-adjoint scalar amplitudes that has minimal rank can be constructed if a specific condition on the masses, given by the equation $\operatorname{det} \mathcal{A}^{\phi^{3}}[\alpha \mid \beta]=0$ is met. We will call this the spectral condition. The null vectors of this matrix will then give rise to massive BCJ relations. On the basis of this observation, we propose the following:

Conjecture: the KLT prescription for double-copying models with massive states generates physical amplitudes without spurious singularities, and reduces smoothly to the massless double-copy in an appropriate $m \rightarrow 0$ decoupling limit, if the associated bi-adjoint scalar matrix has minimal rank.

In this section we will illustrate the consequences of imposing these conditions on models at $n=4$ and $n=5$. We will see how this alternative construction has both a commuting decoupling limit and the absence of spurious singularities, providing evidence in support of our conjecture above.

\subsection{4-point spectral condition}

We will begin with a model that has a more general spectrum of massive or massless states. We denote the external states $m_{i}$ and the intermediate masses being exchanged on a factorization channel as $m_{i j}$. The only assumption we will make is the existence of a BCJ representation of the form

$$
\mathcal{A}_{4}\left(1^{a_{1}}, 2^{a_{2}}, 3^{a_{3}}, 4^{a_{4}}\right)=\frac{c_{12} n_{12}}{s_{12}+m_{12}^{2}}+\frac{c_{13} n_{13}}{s_{13}+m_{13}^{2}}+\frac{c_{14} n_{14}}{s_{14}+m_{14}^{2}} .
$$

Implicitly built into this expression is the assumption that only states with mass $m_{12}^{2}$ are exchanged in the $s_{12}$-channel and so forth. This is not completely general and an interesting open problem is to construct an appropriate generalization of the BCJ form for models with multiple mass states exchanged in a single channel. We now choose a DDM basis $([1234],[1324])$, in which the matrix of bi-adjoint scalar amplitudes is

$$
\mathcal{A}_{4}^{\phi^{3}}[\alpha \mid \beta]=\left(\begin{array}{cc}
\frac{1}{s_{12}+m_{12}^{2}}+\frac{1}{s_{14}+m_{14}^{2}} & -\frac{1}{s_{14}+m_{14}^{2}} \\
-\frac{1}{s_{14}+m_{14}^{2}} & \frac{1}{s_{13}+m_{13}^{2}}+\frac{1}{s_{14}+m_{14}^{2}}
\end{array}\right) .
$$

\footnotetext{
${ }^{11}$ In this work we only consider states that transform in the adjoint representation. The value $(n-3)$ ! for the minimal rank may be modified if particles in other representations are present.
} 
Taking the determinant, gives

$$
\operatorname{det} \mathcal{A}_{4}^{\phi^{3}}=\frac{m_{12}^{2}+m_{13}^{2}+m_{14}^{2}-m_{1}^{2}-m_{2}^{2}-m_{3}^{2}-m_{4}^{2}}{\left(s_{12}+m_{12}^{2}\right)\left(s_{13}+m_{13}^{2}\right)\left(s_{14}+m_{14}^{2}\right)} .
$$

Clearly $\mathcal{A}^{\phi^{3}}[\alpha \mid \beta]$ is full-rank and non-singular, i.e. $\operatorname{det} \mathcal{A}^{\phi^{3}}[\alpha \mid \beta]$ does not vanish, for generic mass spectra. In keeping with our conjecture, we want to reduce the rank of $\mathcal{A}^{\phi^{3}}[\alpha \mid \beta]$ to $(4-3) !=1$, which is the minimal rank at 4 -point order. This is achieved by imposing the following condition on the mass spectrum of the theory,

$$
m_{12}^{2}+m_{13}^{2}+m_{14}^{2}=m_{1}^{2}+m_{2}^{2}+m_{3}^{2}+m_{4}^{2} .
$$

This is the 4-point spectral condition. It is interesting to note that the spectrum of massive Yang-Mills does not satisfy this condition. We will see later that this is what led the double-copy and decoupling limit to fail to commute when studying massive (Yang-Mills) ${ }^{2}$.

On imposing the spectral condition, $\mathcal{A}^{\phi^{3}}[\alpha \mid \beta]$ becomes singular and is no longer invertible. As a result, we must eliminate one row and one column to produce an invertible matrix of bi-adjoint scalar amplitudes. This is consistent only if all such choices give the same result. For example, we could remove the second row and second column, the resulting KLT formula is then

$$
\mathcal{M}_{4}(1,2,3,4)=-\frac{\left(s_{12}+m_{12}^{2}\right)\left(s_{14}+m_{14}^{2}\right)}{s_{13}+\left(m_{1}^{2}+m_{2}^{2}+m_{3}^{2}+m_{4}^{2}-m_{12}^{2}-m_{14}^{2}\right)} \mathcal{A}_{4}[1,2,3,4]^{2} .
$$

If however, we choose to eliminate the second row and the first column, we find

$$
\mathcal{M}_{4}(1,2,3,4)=-\left(s_{14}+m_{14}^{2}\right) \mathcal{A}_{4}[1,2,3,4] \mathcal{A}_{4}[1,3,2,4] .
$$

Equating these formulae constructs a massive version of the fundamental BCJ relation

$$
\left(s_{12}+m_{12}^{2}\right) \mathcal{A}_{4}[1,2,3,4]=\left(s_{13}+m_{13}^{2}\right) \mathcal{A}_{4}[1,3,2,4],
$$

where we have used the spectral condition to rewrite the relation in a more compact form.

As we prove in appendix $\mathrm{F}$, an equivalent way to derive the massive BCJ relation is by studying the null vector of $\mathcal{A}^{\phi^{3}}[\alpha \mid \beta]$ which is

$$
\vec{n}=\left(\begin{array}{c}
-s_{12}-m_{12}^{2} \\
s_{13}+m_{13}^{2}
\end{array}\right)
$$

Setting the dot product of this vector with the DDM basis to zero then gives the BCJ relation,

$$
\vec{n} \cdot\left(\mathcal{A}_{5}[1234] \quad \mathcal{A}_{5}[1324]\right)=\left(s_{12}+m_{12}^{2}\right) \mathcal{A}_{4}[1,2,3,4]-\left(s_{13}+m_{13}^{2}\right) \mathcal{A}_{4}[1,3,2,4]=0 .
$$

We are now in a position to study the singularity structure of the KLT formula (4.5). The first aspect of the formula that we note is the absence of spurious poles, i.e. all poles are 
at physical locations. To ensure locality, we can study the amplitude in the neighbourhood of its three physical poles. For example,

$$
\begin{aligned}
\operatorname{Res}_{s_{12}=-m_{12}^{2}} \mathcal{M}_{4}(1,2,3,4) & =-\frac{\left(s_{14}+m_{14}^{2}\right)}{s_{13}+m_{13}^{2}} \mathcal{A}_{4}\left[1,2,-P_{12}\right]^{2} \mathcal{A}_{3}\left[P_{12}, 3,4\right]^{2} \\
& =\mathcal{A}_{4}\left[1,2,-P_{12}\right]^{2} \mathcal{A}_{3}\left[P_{12}, 3,4\right]^{2} \\
& =\mathcal{M}_{3}\left(1,2,-P_{12}\right) \mathcal{M}_{3}\left(P_{12}, 3,4\right)
\end{aligned}
$$

where we have used $s_{13}+m_{13}^{2}=-s_{14}-m_{14}^{2}$ on the $s_{12}$ pole. Thus the amplitude factorizes correctly on the $s_{12}$ pole. Factorization on the $s_{13}$ and $s_{14}$ pole follow in a similar manner.

It is easy to see that these forms of the massive BCJ relations and KLT formula smoothly reduce to the massless ones when all external and intermediate masses, $m_{i}$ and $m_{i j}$ are taken to zero. As a result, this version of the massive double-copy does commute with the decoupling limit. Thus for any pair of massive BCJ-compatible theories $A^{(m)}$ and $B^{(m)}$ that satisfy the spectral condition, one can construct a local theory,

$$
C^{(m)}=A^{(m)} \otimes_{m} B^{(m)},
$$

where $\otimes_{m}$ is our conjectured massive KLT formalism. This will reduce in the decoupling limit to

$$
\lim _{m \rightarrow 0} C^{(m)}=\lim _{m \rightarrow 0}\left(A^{(m)} \otimes_{m} B^{(m)}\right)=\left(\lim _{m \rightarrow 0} A^{(m)}\right) \otimes\left(\lim _{m \rightarrow 0} B^{(m)}\right),
$$

where $\otimes$ denotes the massless KLT double-copy.

As we saw in section 2.1, the massive KLT and massive BCJ double copies are equivalent. Let us now understand our conjecture from the perspective of the BCJ double-copy. We begin by considering the effect of a generalized gauge transformation on the BCJ representation. The amplitude is invariant under the following replacements

$$
\begin{aligned}
& n_{12} \rightarrow n_{12}+\left(s_{12}+m_{12}^{2}\right) \Delta \\
& n_{13} \rightarrow n_{13}+\left(s_{13}+m_{13}^{2}\right) \Delta \\
& n_{14} \rightarrow n_{14}+\left(s_{14}+m_{14}^{2}\right) \Delta,
\end{aligned}
$$

for any function $\Delta$. Putting these together we find the kinematic Jacobi sum of numerators transforms as

$$
n_{12}+n_{13}+n_{14} \rightarrow n_{12}+n_{13}+n_{14}+\left(m_{12}^{2}+m_{13}^{2}+m_{14}^{2}-m_{1}^{2}-m_{2}^{2}-m_{3}^{2}-m_{4}^{2}\right) \Delta .
$$

If the spectral condition is not satisfied then we can always find a generalized gauge in which the numerators satisfy color-kinematics duality by using,

$$
\Delta=-\frac{n_{12}+n_{13}+n_{14}}{\left(m_{12}^{2}+m_{13}^{2}+m_{14}^{2}-m_{1}^{2}-m_{2}^{2}-m_{3}^{2}-m_{4}^{2}\right)} .
$$

If the spectral condition is satisfied, however, then there is no choice of $\Delta$ that can construct numerators that satisfy the kinematic Jacobi relations from ones that do not. 
Hence the existence of kinematic Jacobi-satisfying numerators is a non-trivial constraint on the space of BCJ-like models, equivalent to imposing the massive fundamental BCJ relations.

At 4-point, we saw that there is a well-chosen BCJ basis in which the KLT kernel is polynomial, and therefore together with the discussion in section 2.2, the resulting formula defines an amplitude with only physical singularitites. The BCJ version of this statement is that if the spectral condition is satisfied, and there exist color-kinematics duality satisfying numerators, then the BCJ double-copy is free of spurious singularities.

It is clear that a model with a uniform mass spectrum like massive Yang-Mills could only satisfy the 4-point spectral condition if all of the states have zero mass. For more complicated models, with states of multiple masses, the constraints are very restrictive. We will now illustrate these constraints with a few examples.

Example 1: Compton scattering. Consider a model such as Yang-Mills minimally coupled to a complex adjoint scalar with mass $m \neq 0$. There are three factorization channels contributing to the Compton amplitude $g+\phi \rightarrow g+\phi$ :
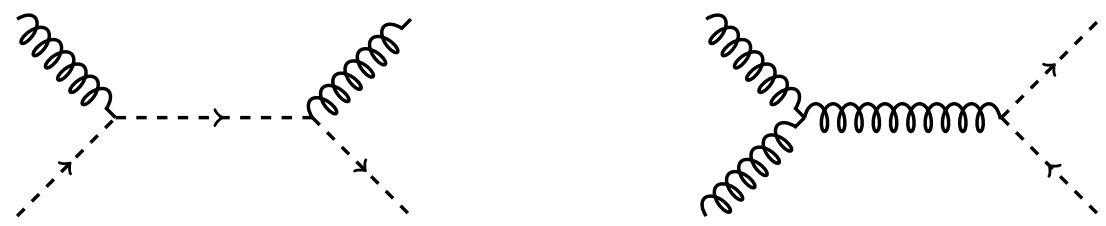

The first diagram contributes twice, corresponding to exchanging the labels on the gluons. Here the spectral condition is satisfied since for the external states

$$
m_{1}^{2}+m_{2}^{2}+m_{3}^{2}+m_{4}^{2}=2 m^{2},
$$

while for the internal states

$$
m_{12}^{2}+m_{13}^{2}+m_{14}^{2}=2 m^{2} .
$$

We must keep in mind that the spectral condition is only a conjectured necessary condition for the existence of a local double-copy, not a sufficient one. For a theory to produce a local double-copy, it must also satisfy the BCJ relations. The fact that a sensible double-copy of Compton scattering amplitudes can be defined only if the theory satisfies the massive BCJ relations (4.7) was first observed in [12].

Explicitly the color-ordered amplitudes [11]

$$
\begin{aligned}
& \mathcal{A}_{4}\left[1_{\phi}, 2_{g}^{+}, 3_{g}^{-}, 4_{\bar{\phi}}\right]=-\frac{\left\langle 3\left|p_{1}\right| 2\right]^{2}}{s_{23}\left(s_{12}+m^{2}\right)} \\
& \mathcal{A}_{4}\left[1_{\phi}, 3_{g}^{-}, 2_{g}^{+}, 4_{\bar{\phi}}\right]=-\frac{\left\langle 3\left|p_{1}\right| 2\right]^{2}}{s_{23}\left(s_{13}+m^{2}\right)},
\end{aligned}
$$

satisfy the massive BCJ relation (4.7). According to our conjecture the double-copy and the massless limit should commute in such a case. Indeed, taking the massive double-copy and then the massless limit

$$
\mathcal{M}_{4}^{m \neq 0}\left(1_{\phi}, 2_{h}^{+}, 3_{h}^{-}, 4_{\bar{\phi}}\right)=\frac{\left\langle 3\left|p_{1}\right| 2\right]^{4}}{\left(s_{12}+m^{2}\right)\left(s_{13}+m^{2}\right)} \stackrel{m=0}{\longrightarrow} \frac{\left\langle 3\left|p_{1}\right| 2\right]^{4}}{s_{12} s_{13}},
$$


compared to taking the massless limit and then the double-copy

$$
s_{14} \mathcal{A}_{4}^{m=0}\left[1_{\phi}, 2_{g}^{+}, 3_{g}^{-}, 4_{\bar{\phi}}\right] \mathcal{A}_{4}^{m=0}\left[1_{\phi}, 3_{g}^{-}, 2_{g}^{+}, 4_{\phi}\right]=\frac{\left\langle 3\left|p_{1}\right| 2\right]^{4}}{s_{12} s_{13}},
$$

gives the same result.

Example 2: Bhabha scattering. In the same model as the previous example we can consider Bhabha scattering $\phi+\bar{\phi} \rightarrow \phi+\bar{\phi}$ which has two contributing factorization channels related by relabelling:

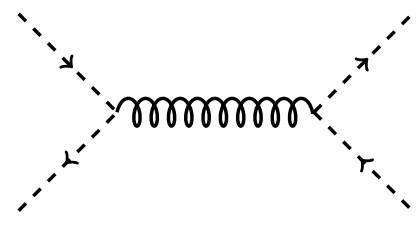

Here the spectral condition is not satisfied since for the external states

$$
m_{1}^{2}+m_{2}^{2}+m_{3}^{2}+m_{4}^{2}=4 m^{2}
$$

while for the internal states

$$
m_{12}^{2}+m_{13}^{2}+m_{14}^{2}=0 .
$$

Since the spectral condition is not satisfied, there are no associated fundamental BCJ conditions. Similar to the 4-point massive Yang-Mills calculation, we can find color-kinematics duality satisfying numerators and take a massive double-copy, but such an amplitude should not have a smooth $m \rightarrow 0$ limit. It is instructive to see this explicitly. We begin with the tree-amplitude calculated using ordinary Feynman rules for a minimally coupled scalar

$$
\mathcal{A}_{4}\left(1_{\phi}^{a_{1}}, 2_{\bar{\phi}}^{a_{2}}, 3_{\phi}^{a_{3}}, 4_{\bar{\phi}}^{a_{4}}\right)=c_{12} \frac{s_{13}-s_{14}}{s_{12}}+c_{14} \frac{s_{12}-s_{13}}{s_{14}} .
$$

The corresponding BCJ numerators,

$$
\begin{aligned}
& n_{12}=s_{13}-s_{14} \\
& n_{13}=0 \\
& n_{14}=s_{12}-s_{13},
\end{aligned}
$$

do not satisfy the kinematic Jacobi relation. We can construct numerators which do, however, by making a generalized gauge transformation

$$
\begin{aligned}
& \hat{n}_{12}=s_{13}-s_{14}+\frac{1}{4 m^{2}} s_{12}\left(s_{12}-s_{14}\right) \\
& \hat{n}_{13}=\frac{1}{4 m^{2}} s_{13}\left(s_{12}-s_{14}\right) \\
& \hat{n}_{14}=s_{12}-s_{13}+\frac{1}{4 m^{2}} s_{14}\left(s_{12}-s_{14}\right) .
\end{aligned}
$$


Forming the massive BCJ double-copy, we find

$$
\begin{aligned}
\mathcal{M}_{4}\left(1_{\phi}, 2_{\bar{\phi}}, 3_{\phi}, 4_{\bar{\phi}}\right)= & \frac{\left(s_{13}-s_{14}\right)^{2}}{s_{12}}+\frac{\left(s_{12}-s_{13}\right)^{2}}{s_{14}} \\
& +4 m^{2}+4 s_{12}+2 s_{13}+\frac{1}{4 m^{2}}\left(4 s_{12}^{2}+4 s_{12} s_{13}+s_{13}^{2}\right) .
\end{aligned}
$$

While this is a perfectly physical scattering amplitude, the massive double-copy has generated a contact contribution corresponding to a local operator of the form $\frac{1}{m^{2} M_{p}^{2}}(\partial \phi)^{4}$, which diverges as $m \rightarrow 0$.

Example 3: Kaluza-Klein theory. An important class of examples arises from the dimensional reduction of the massless KLT relations in higher dimensions, some of which have already been discussed in [13-16, 20]. This has the effect of generating a Kaluza-Klein tower of states and vertices that conserve Kaluza-Klein number. This conservation law manifests as a conservation of mass at each vertex. For concreteness, consider a $d=5$ scalar model compactified on $\mathbb{R}^{4} \times S^{1}$, and take for example the scattering process $1+2 \rightarrow 3+4$, where all of the external states are right-moving $\left(p_{i}^{4}=+m_{i}\right)$ states. At the vertices the masses satisfy the sum rules

$$
\begin{aligned}
& m_{1}+m_{2}=m_{12} \\
& m_{1}-m_{3}=m_{13} \\
& m_{1}-m_{4}=m_{14} \\
& m_{1}+m_{2}=m_{3}+m_{4} .
\end{aligned}
$$

In this case as well, the spectral condition holds with no further constraints,

$$
\begin{aligned}
\Rightarrow m_{12}^{2}+m_{13}^{2}+m_{14}^{2} & =3 m_{1}^{2}+m_{2}^{2}+m_{3}^{2}+m_{4}^{2}+2 m_{1} m_{2}-2 m_{1} m_{4}-2 m_{1} m_{3} \\
& =3 m_{1}^{2}+m_{2}^{2}+m_{3}^{2}+m_{4}^{2}-2 m_{1}^{2} \\
& =m_{1}^{2}+m_{2}^{2}+m_{3}^{2}+m_{4}^{2} .
\end{aligned}
$$

Thus any theory that arises as a dimensional reduction of a massless BCJ-compatible theory will automatically satisfy the spectral condition and thus it will give a local double-copy. Such a model gives a complete example, for which every scattering amplitude satisfies the spectral constraints, and moreover, if the higher-dimensional model satisfies the massless BCJ relations then so too will the lower-dimensional Kaluza-Klein model. We leave as future work the problem of determining if there are additional complete examples which are not obtained by dimensional reduction.

\subsection{5 -point spectral conditions}

Locality places the strongest constraints on the massive double-copy. As was exemplified in section 3.4, demanding the existence of color-kinematics duality satisfying 5-point numerators is not a strong enough condition to ensure locality of double-copied 5-point amplitudes. A natural question is what conditions need to be satisfied at 5-point in order for the resulting double-copied amplitude to be local. 
We set the calculation up in a manner similar to the 4-point case. We assume the existence of a BCJ representation and allow for general external and intermediate masses, $m_{i}$ and $m_{i j}$ respectively. Here the masses $m_{i j}$ are exchanged on the $i j$ 2-particle channel. We can then write down a bi-adjoint scalar matrix (B.3) where each propagator $s_{i j}+m^{2}$ is now replaced by $s_{i j}+m_{i j}^{2}$.

We know that 5-point amplitudes need to factorize on 2-particle channels to give 4point amplitudes. At 4-point, we saw that locality is only ensured by requiring that the matrix of bi-adjoint scalar amplitudes is singular. This is achieved via the so-called spectral condition (4.4). On demanding that this condition is satisfied on every possible 4-point amplitude that could result on a factorization channel, we come up with the following set of conditions,

$$
m_{i j}^{2}+m_{i k}^{2}+m_{j k}^{2}=m_{i}^{2}+m_{j}^{2}+m_{k}^{2}+m_{p q}^{2}
$$

for each triplet $i, j, k$ and where $p, q$ are the leftover elements in $\{1,2,3,4,5\}$. There are ${ }^{5} C_{3}=10$ such relations, but they are not all independent. We can reduce them to 5 independent conditions,

$$
\begin{aligned}
& m_{15}^{2}=2 m_{1}^{2}-m_{12}^{2}-m_{13}^{2}-m_{14}^{2}+m_{2}^{2}+m_{3}^{2}+m_{4}^{2}+m_{5}^{2} \\
& m_{25}^{2}=m_{1}^{2}-m_{12}^{2}+2 m_{2}^{2}-m_{23}^{2}-m_{24}^{2}+m_{3}^{2}+m_{4}^{2}+m_{5}^{2} \\
& m_{34}^{2}=2 m_{1}^{2}-m_{12}^{2}-m_{13}^{2}-m_{14}^{2}+2 m_{2}^{2}-m_{23}^{2}-m_{24}^{2}+2 m_{3}^{2}+2 m_{4}^{2}+m_{5}^{2} \\
& m_{35}^{2}=-m_{1}^{2}+m_{12}^{2}+m_{14}^{2}-m_{2}^{2}+m_{24}^{2}-m_{4}^{2} \\
& m_{45}^{2}=-m_{1}^{2}+m_{12}^{2}+m_{13}^{2}-m_{2}^{2}+m_{23}^{2}-m_{3}^{2} .
\end{aligned}
$$

We will refer to these as the 5-point spectral conditions. These conditions indeed make the bi-adjoint scalar matrix singular. Further, they reduce the rank of the $(n-2) ! \times(n-2) !=$ $6 \times 6$ matrix from full-rank to minimal rank, $(n-3) !=2$.

As we show in appendix $\mathrm{F}$, the null vectors of the bi-adjoint scalar matrix give us the 5-point massive BCJ relations,

$$
\begin{aligned}
\mathcal{A}_{5}[13452]= & \left(-\frac{m_{12}^{2}+s_{12}}{m_{34}^{2}+s_{34}}+\frac{m_{35}^{2}+s_{35}}{m_{34}^{2}+s_{34}}\right) \mathcal{A}_{5}[13542]+\left(\frac{m_{14}^{2}+s_{14}}{m_{34}^{2}+s_{34}}\right) \mathcal{A}_{5}[13524], \\
\mathcal{A}_{5}[13425]= & \left(\frac{\left(m_{12}^{2}+s_{12}\right)\left(m_{45}^{2}+s_{45}\right)}{\left(m_{15}^{2}+s_{15}\right)\left(m_{34}^{2}+s_{34}\right)}\right) \mathcal{A}_{5}[13542] \\
& +\left(\frac{m_{14}^{2}+s_{14}}{m_{15}^{2}+s_{15}}-\frac{\left(m_{12}^{2}+s_{12}\right)\left(m_{14}^{2}+s_{14}\right)}{\left(m_{15}^{2}+s_{15}\right)\left(m_{34}^{2}+s_{34}\right)}\right) \mathcal{A}_{5}[13524], \\
\mathcal{A}_{5}[13245]= & \left(\frac{m_{12}^{2}+s_{12}}{m_{15}^{2}+s_{15}}-\frac{\left(m_{12}^{2}+s_{12}\right)\left(m_{14}^{2}+s_{14}\right)}{\left(m_{15}^{2}+s_{15}\right)\left(m_{23}^{2}+s_{23}\right)}\right) \mathcal{A}_{5}[13542] \\
& +\left(\frac{\left(m_{14}^{2}+s_{14}\right)\left(m_{25}^{2}+s_{25}\right)}{\left(m_{15}^{2}+s_{15}\right)\left(m_{23}^{2}+s_{23}\right)}\right) \mathcal{A}_{5}[13524], \\
\mathcal{A}_{5}[13254]= & \left(-\frac{m_{12}^{2}+s_{12}}{m_{23}^{2}+s_{23}}\right) \mathcal{A}_{5}[13542]+\left(-\frac{m_{12}^{2}+s_{12}}{m_{23}^{2}+s_{23}}-\frac{m_{24}^{2}+s_{24}}{m_{23}^{2}+s_{23}}\right) \mathcal{A}_{5}[13524],
\end{aligned}
$$


with the understanding that $m_{15}, m_{25}, m_{34}, m_{35}$ and $m_{45}$ are given by the spectral conditions (4.30).

Choosing any $2 \times 2$ submatrix $\mathcal{A}^{\phi^{3}}[\alpha \mid \beta]$ of the bi-adjoint scalar matrix is now invertible and can be used to define a local double-copy. For example,

$$
\mathcal{A}_{5}^{A \otimes B}(12345)=\sum_{\alpha, \beta=[13542],[13524]} \mathcal{A}_{5}^{A}[\alpha] \mathcal{A}^{\phi^{3}}[\alpha \mid \beta]^{-1} \mathcal{A}_{5}^{A}[\beta]
$$

where

$$
\mathcal{A}^{\phi^{3}}[\alpha \mid \beta]=\left(\begin{array}{cc}
\frac{1}{D_{1}}+\frac{1}{D_{12}}+\frac{1}{D_{2}}+\frac{1}{D_{6}}+\frac{1}{D_{9}} & -\frac{1}{D_{12}}-\frac{1}{D_{9}} \\
-\frac{1}{D_{12}}+\frac{1}{D_{9}} & \frac{1}{D_{12}}+\frac{1}{D_{3}}+\frac{1}{D_{4}}+\frac{1}{D_{5}}+\frac{1}{D_{9}}
\end{array}\right),
$$

and $D_{i}$ are as defined in appendix B.

To explicitly see that the resulting amplitude is local, we perform the following tests. First, we look at the denominator of the resulting KLT formula,

$$
\left(s_{15}+m_{15}^{2}\right)\left(m_{23}^{2}+s_{23}\right)\left(s_{34}+m_{34}^{2}\right),
$$

again with the understanding that $m_{i j}$ satisfy the spectral conditions (4.30) and note that the KLT formula only has poles in physical locations.

Second, we must check that $\mathcal{A}_{5}^{A \otimes B}(12345)$ factorizes correctly on all poles. Let us look at an example. Consider the pole $s_{23} \rightarrow-m_{23}^{2}$,

$$
\begin{aligned}
\operatorname{Res}_{s_{23}=-m_{23}^{2}} \mathcal{A}_{5}^{A \otimes B}(12345)= & \frac{\left(m_{14}^{2}+s_{14}\right)\left(m_{12}^{2}+m_{13}^{2}+s_{12}+s_{13}\right)}{\left(s_{15}+m_{15}^{2}\right)}\left[\mathcal{A}_{5}[13542]\left(m_{12}^{2}+s_{12}\right)\right. \\
& \left.+\mathcal{A}_{5}[13524]\left(m_{12}^{2}+m_{24}^{2}+s_{12}+s_{24}\right)\right]^{2}
\end{aligned}
$$

The massive BCJ relation (4.34) tells us that the expression in the square brackets is $\mathcal{A}_{5}[13254]$ which factorizes into $\mathcal{A}_{3}\left[32\left(-P_{23}\right)\right] \times \mathcal{A}_{4}\left[P_{23} 541\right]$ on the pole to give

$$
\begin{aligned}
\operatorname{Res}_{s_{23}=-m_{23}^{2}} \mathcal{A}_{5}^{A \otimes B}(12345) & =\frac{\left(m_{14}^{2}+s_{14}\right)\left(m_{12}^{2}+m_{13}^{2}+s_{12}+s_{13}\right)}{\left(s_{15}+m_{15}^{2}\right)}\left(\mathcal{A}_{3}\left[32\left(-P_{23}\right)\right] \mathcal{A}_{4}\left[P_{23} 541\right]\right)^{2} \\
& =\mathcal{A}_{3}^{A \otimes B}\left(32\left(-P_{23}\right)\right) \times \mathcal{A}_{4}^{A \otimes B}\left(P_{23} 541\right),
\end{aligned}
$$

where we have used the 4-point KLT formula in the last step. Thus the amplitude factorizes correctly on the $s_{23}=-m_{23}^{2}$ pole.

One can proceed in a similar manner (either with or without the help of massive BCJ relations) to determine that the 5-point KLT formula (4.35) factorizes correctly on all poles. Thus, given a theory that satisfies the 5-point spectral conditions, the KLT formula constructs local amplitudes, giving us a sensible definition of the 5-point double-copy. 


\subsection{Non-minimal rank}

There is a new possibility that arises at higher-point which is not present at 4-point. This is the ability to reduce the rank of a bi-adjoint scalar matrix from full-rank $(n-2)$ ! not to minimal rank $(n-3)$ !, but somewhere in between $(n-2)$ ! and $(n-3)$ !. Since this too makes the $(n-2) ! \times(n-2)$ ! matrix singular, one might imagine this to be an alternate approach to the massive double-copy that does not require all four BCJ relations to hold. Indeed such a procedure does not give rise to local amplitudes. Let us understand how this works at 5-point.

By imposing all-but-one of the spectral conditions (4.30), the rank of the 5-point biadjoint scalar matrix reduces from 6 to 4, rather than minimal rank 2. For example, let us choose not to impose the spectral condition on $m_{34}^{2}$. Since the resulting expressions are difficult to manipulate analytically, we proceed in a particular kinematic configuration where all-but-one (let us say $s_{12}$ ) independent Mandelstam variables are fixed.

We can now check the behaviour of the double-copied amplitude as we approach the pole $s_{12}=-m_{12}^{2}$. We want the double-copied amplitude to factorize as,

$$
\underset{s_{12}=-m_{12}^{2}}{\operatorname{Res}} \mathcal{A}_{5}^{A \otimes B}(12345)=\mathcal{A}_{3}^{A \otimes B}\left(12\left(-P_{12}\right)\right) \times \mathcal{A}_{4}^{A \otimes B}\left(P_{12} 345\right) .
$$

We find that this condition is not met unless,

$$
m_{34}^{2}=2 m_{1}^{2}-m_{12}^{2}-m_{13}^{2}-m_{14}^{2}+2 m_{2}^{2}-m_{23}^{2}-m_{24}^{2}+2 m_{3}^{2}+2 m_{4}^{2}+m_{5}^{2},
$$

which is exactly the spectral condition that we left out. Thus, by not imposing all of the BCJ relations, we do not construct local amplitudes.

This supports our conjecture: only by imposing all BCJ relations, i.e. reducing the bi-adjoint scalar matrix to minimal rank, can we construct local amplitudes via the KLT formula.

\section{Discussion}

The proposition of a KLT construction for the double-copy of massive particles opens up many areas of exploration and application. In section 2.2, we noted that our argument for the existence of spurious singularities in the proposed double-copy formula (2.11) does not apply to three-dimensional theories. This suggests that the prescription (2.11) might be healthy in $3 \mathrm{~d}$, despite suffering from spurious singularities in $4 \mathrm{~d}$ and higher, with a possible example being the conjectured double copy construction of topologically massive gravity from topologically massive gauge theory [41].

In section 3, we see that a putative double-copy of massive Yang-Mills is not wellbehaved due to the presence of spurious singularities in the would-be double-copied 5point amplitude. Further work is needed to address the problems brought to light here. For example, one could investigate the possibility of addition of 5-point operators or new degrees of freedom to the massive Yang-Mills EFT to construct a local double-copy.

Another interesting question is what happens when the bi-adjoint Higgs model presented in section 3 is double copied with itself. It has been shown that the high energy 
behaviour of a theory of $\Lambda_{3}$ massive gravity cannot be improved by introducing vector or scalar interactions [42]. Therefore, we expect the double-copy of the bi-adjoint Higgs model to fail. A better understanding of the precise nature of this failure would be interesting.

An important assumption that lead to the derivation of the mass spectral conditions presented in section 4 was that a unique mass is exchanged in each factorization channel. We know that a massless KLT formula can be constructed that allows for the exchange of particles of multiple masses on each channel [22]. It would be interesting to see how this construction generalizes to the case of massive external particles and more general spectra.

In addition, we would like to better understand the landscape of theories that produce a local double-copy. We saw examples of dimensionally reduced BCJ-compatible theories in which the Kaluza-Klein tower of massive states and interactions between them manifestly satisfy the spectral condition and hence result in local double-copied amplitudes. We would like to understand whether there are double-copy-compatible theories that do not result from a dimensional reduction.

Finally, in section 4, we saw that spurious singularities are removed if the spectral conditions and massive BCJ relations are satisfied. However, we know that massive biadjoint scalar theory trivially provides an explicit counter-example to making the converse statement, since it will produce a local, massive double-copy even if the spectral conditions are not satisfied. It is therefore an interesting open problem to determine if there exist further, non-trivial, examples of massive models which double-copy to physical scattering amplitudes but do not satisfy the spectral condition. One pathway to such a construction would be to try and find a model which admits a local, off-shell representation of the kinematic algebra, similar to [43, 44]. Since the numerators of such a model are local by construction, it is clear from the BCJ form of the double-copy that no spurious poles can be generated. Even more interestingly, given such a set of local, kinematic Jacobi satisfying numerators, we can always form a heterotic double-copy with the numerators of a generic, spectral condition violating, massive model. Since the result does not depend on the generalized gauge used for the numerators of the latter, they can always be taken to be the local representation given by Feynman rules, and so even in this case, we see that no spurious poles can be generated. We see then that constructing even a single example of a model with a local, off-shell representation of the kinematic algebra, is sufficient to generate an infinite number of examples of healthy, massive double-copies. We leave this and similar investigations to future work.

\section{Acknowledgments}

We would like to thank James Bonifacio, Clifford Cheung, Henriette Elvang, Kurt Hinterbichler, Henrik Johansson and Andrew Tolley for useful discussions. The work of CRTJ was supported by a Rackham Predoctoral Fellowship from the University of Michigan. The work of SP was supported in part by the US Department of Energy under Grant No. DE-SC0007859. We would also like to acknowledge the TASI 2019 summer school, which brought us together and played host to preliminary discussions. 


\section{A Massless limits of massive theories}

In this appendix, we will review taking massless limits of massive theories. If the massless limit in the Lagrangian of a higher spin $(\geq 1)$ particle is taken by just setting $m \rightarrow 0$, degrees of freedom can be lost causing the limit to be discontinuous. Here we show how to take the massless limit in a continuous way that preserves the number of degrees of freedom. More extensive reviews can be found in [34, 45].

Example 1: massive photon. To start with, we examine the Lagrangian of a massive photon with a quartic interaction term,

$$
\mathcal{L}=-\frac{1}{4} F_{\mu \nu} F^{\mu \nu}-\frac{1}{2} m^{2} A_{\mu} A^{\mu}+g\left(A_{\mu} A^{\mu}\right)^{2} .
$$

The mass term and interaction term break the $U(1)$ gauge symmetry $\delta A_{\mu}=\partial_{\mu} \Lambda$ that a massless photon would have. If we were to try to take the massless limit by setting $g, m \rightarrow 0$, the limit would not be continuous, as in 4 dimensions a massive photon has 3 degrees of freedom regardless of how small the mass, while a massless photon has 2 degrees of freedom.

In order to properly take the massless limit, the limit must be taken in a way that preserves the number of degrees of freedom. One way to explicitly see how the discontinuity arises is by using the Stückelberg trick. This involves introducing new fields in a way that makes the theory gauge invariant, but is still dynamically equivalent to the original theory. To do this, we make a replacement of the field patterned after the $U(1)$ gauge symmetry enjoyed by a massless photon:

$$
A_{\mu} \rightarrow A_{\mu}+\frac{1}{m} \partial_{\mu} \phi
$$

This gives an action,

$\mathcal{L}=-\frac{1}{4} F_{\mu \nu} F^{\mu \nu}-\frac{1}{2} m^{2}\left(A_{\mu}+\frac{1}{m} \partial_{\mu} \phi\right)\left(A^{\mu}+\frac{1}{m} \partial^{\mu} \phi\right)+g\left(\left(A_{\mu}+\frac{1}{m} \partial_{\mu} \phi\right)\left(A^{\mu}+\frac{1}{m} \partial^{\mu} \phi\right)\right)^{2}$,

which is gauge invariant under the transformations:

$$
\delta A_{\mu}=\partial_{\mu} \lambda, \quad \delta \phi=-m \lambda .
$$

This action, although it contains more fields, is completely equivalent to (A.1) since (A.3) is gauge invariant, and we can always choose unitary gauge, $\phi=0$ to recover the original action. Expanding the action, we find:

$$
\begin{aligned}
\mathcal{L}= & -\frac{1}{4} F_{\mu \nu} F^{\mu \nu}-\frac{1}{2} m^{2} A_{\mu} A^{\mu}-m A_{\mu} \partial^{\mu} \phi A^{\mu}-\frac{1}{2} \partial_{\mu} \phi \partial^{\mu} \phi+g\left(A_{\mu} A^{\mu}\right)^{2}+4 \frac{g}{m} A_{\mu} A^{\mu} A_{\nu} \partial^{\nu} \phi \\
& +\frac{g}{m^{2}}\left(4\left(A_{\mu} \partial^{\mu} \phi\right)^{2}+2 A_{\mu} A^{\mu} \partial_{\nu} \phi \partial^{\nu} \phi\right)+4 \frac{g}{m^{3}} A_{\mu} \partial^{\mu} \phi \partial_{\nu} \phi \partial^{\nu} \phi+\frac{g}{m^{4}}\left(\partial_{\mu} \phi \partial^{\mu} \phi\right)^{2} .
\end{aligned}
$$

Now, when taking the massless limit, the lowest energy scale suppressing the interaction terms is $\Lambda=\left(m^{4} / g\right)^{1 / 4}$. If we now take the massless decoupling limit,

$$
g, m \rightarrow 0, \quad \Lambda=\left(\frac{m^{4}}{g}\right)^{1 / 4}, A_{\mu}, \phi \text { fixed }
$$


we find all the interaction terms vanish except for the scalar self-interaction terms, giving the Lagrangian,

$$
\mathcal{L}=-\frac{1}{4} F_{\mu \nu} F^{\mu \nu}-\frac{1}{2} \partial_{\mu} \phi \partial^{\mu} \phi+\frac{1}{\Lambda^{4}}\left(\partial_{\mu} \phi \partial^{\mu} \phi\right)^{2},
$$

the number of degrees of freedom is preserved, two in the form of a massless vector and one in the form of a scalar with a quartic self-interaction term, and the action is gauge invariant

$$
\delta A_{\mu}=\partial_{\mu} \lambda, \quad \delta \phi=0 .
$$

In taking the massless limit this way, it is obvious that the limit is smooth and that the massless limit of a massive vector is not just a massless vector, but is a massless vector plus a scalar.

Example 2: linearized massive graviton. The linearized Lagrangian for ghost-free massive gravity is given by the action:

$$
\mathcal{L}_{m}=-\frac{1}{2} \partial_{\alpha} h_{\mu \nu} \partial^{\alpha} h^{\mu \nu}+\partial_{\alpha} h_{\mu \nu} \partial^{\nu} h^{\mu \alpha}-\partial_{\mu} h \partial_{\nu} h^{\mu \nu}+\frac{1}{2} \partial_{\mu} h \partial^{\mu} h-\frac{1}{2} m^{2}\left(h_{\mu \nu} h^{\mu \nu}-h^{2}\right) .
$$

One can easily see that the linearized Lagrangian for general relativity can be recovered by setting $m=0$. However, when the massless limit is taken this way, just as in the massive vector case, degrees of freedom are lost as a massive graviton has 5 , while a massless graviton only has 2 degrees of freedom. The gauge invariance that kills the extra degrees of freedom only appears when the mass is exactly zero. We can use the Stückelberg trick to take the massless limit in a way that preserves the number of degrees of freedom. To do this we make a replacement of the field patterned after the linearized diffeomorphism gauge symmetry:

$$
h_{\mu \nu} \rightarrow h_{\mu \nu}+\frac{1}{m} \partial_{\mu} A_{\nu}+\frac{1}{m} \partial_{\nu} A_{\mu}+\frac{1}{m^{2}} \partial_{\mu} \partial_{\nu} \phi
$$

giving the Lagrangian:

$$
\mathcal{L}=\mathcal{L}_{m}-\frac{1}{2} F_{\mu \nu} F^{\mu \nu}-2 m\left(h_{\mu \nu} \partial^{\mu} A^{\nu}-h \partial_{\mu} A^{\mu}\right)-2\left(h_{\mu \nu} \partial^{\mu} \partial^{\nu} \phi-h \partial^{2} \phi\right) .
$$

This action is invariant under the gauge transformations:

$$
\begin{aligned}
\delta h_{\mu \nu} & =\partial_{\mu} \xi_{\nu}+\partial_{\nu} \xi_{\mu}, \quad \delta A_{\mu}=-m \xi_{\mu} \\
\delta A_{\mu} & =\partial_{\mu} \lambda, \quad \delta \phi=-m \lambda
\end{aligned}
$$

This Lagrangian is dynamically equivalent to (A.9) since it is gauge invariant and we can always choose the gauge $A_{\mu}=0, \phi=0$ to recover (A.9). Now if we take the massless limit, the Lagrangian is

$$
\mathcal{L}=\mathcal{L}_{m=0}-\frac{1}{2} F_{\mu \nu} F^{\mu \nu}-2\left(h_{\mu \nu} \partial^{\mu} \partial^{\nu} \phi-h \partial^{2} \phi\right)
$$


and the gauge transformations are

$$
\begin{aligned}
\delta h_{\mu \nu} & =\partial_{\mu} \xi_{\nu}+\partial_{\nu} \xi_{\mu} \\
\delta A_{\mu} & =\partial_{\mu} \lambda \\
\delta \phi & =0 .
\end{aligned}
$$

We find the degrees of freedom break up into 2 tensor modes, 2 vector modes, and 1 scalar mode. So the massless limit of massive gravity is not massless gravity, but massless gravity plus extra degrees of freedom. The tensor modes and scalar mode are coupled. They can be decoupled using a field redefinition, $h_{\mu \nu} \rightarrow h_{\mu \nu}+\pi \eta_{\mu \nu}$. However, if the graviton is coupled to a stress-energy tensor this leads to non-minimal coupling to the stress energy tensor, the so-called vDVZ discontinuity [46].

Example 3: massive Yang-Mills. The Stückelberg trick can be extended to nonAbelian theories as well. To demonstrate, we examine massive Yang-Mills,

$$
\mathcal{L}=-\frac{1}{4}\left(F_{\mu \nu}^{a}\right)^{2}-\frac{1}{2} m^{2} A_{\mu}^{a} A^{a \mu},
$$

where

$$
F_{\mu \nu}^{a} \equiv \partial_{[\mu} A_{\nu]}^{a}+g f^{a b c} A_{\mu}^{b} A_{\nu}^{c} .
$$

Without the mass term, the action would be gauge invariant under: $A_{\mu} \rightarrow R A_{\mu} R^{\dagger}+$ $R \partial_{\mu} R^{\dagger}$, where $R=e^{-i \alpha_{a} T^{a}}, T^{a}$ are the generators of the gauge group, and $\alpha_{a}(x)$ are gauge parameters, but the mass term breaks this gauge symmetry. Just as in the previous examples, we can make a field replacement patterned after the gauge symmetry to create a Lagrangian that is gauge invariant:

$$
A_{\mu} \rightarrow U A_{\mu} U^{\dagger}+U \partial_{\mu} U^{\dagger}
$$

where $U=e^{-i \frac{g}{m} \pi_{a} T^{a}}$ and $\pi_{a}(x)$ are scalar fields. This can give interactions that go like:

$$
\sim g \partial A^{3} \sim g^{2} A^{4}, \quad \sim g^{2}\left(\frac{g}{m}\right)^{n-2} A^{2} \pi^{n} \sim g\left(\frac{g}{m}\right)^{n-2} \partial A \pi^{n} \sim\left(\frac{g}{m}\right)^{n-2} \partial^{2} \pi^{n} .
$$

For $g<1$, the lowest energy scale suppressing the interaction terms is given by $\sim \frac{m}{g}$. We can take the decoupling limit by sending:

$$
g, m \rightarrow 0, \quad \frac{g}{m}, A_{\mu}, \pi \text { fixed. }
$$

In this limit, the only terms that survive are the pure scalar interactions, given by the Lagrangian

$$
\mathcal{L}=\frac{m^{2}}{g^{2}} \operatorname{Tr}\left(\partial_{\mu} U^{\dagger} \partial^{\mu} U\right)
$$

and the free vector fields. 
Example 4: non-linear massive gravity. As a final example, we will examine the massless decoupling limit of full non-linear massive gravity. Using the ghost-free potential for massive gravity, given by the action in (3.33), and assuming all fields are canonically normalized, one finds generic terms with $n_{h}$ powers of $h_{\mu \nu}, n_{A}$ powers of $A_{\mu}$, and $n_{\phi}$ powers of $\phi$ is given by

$$
\sim \Lambda_{\lambda}^{2-n_{h}-2 n_{A}-3 n_{\phi}} h^{n_{h}}(\partial A)^{n_{A}}\left(\partial^{2} \phi\right)^{n_{\phi}},
$$

where the term is suppressed by the scale

$$
\Lambda_{\lambda}=\left(M_{P} m^{\lambda-1}\right)^{1 / \lambda}, \quad \lambda=\frac{3 n_{\phi}+2 n_{A}+n_{h}-4}{n_{\phi}+n_{A}+n_{h}-2} .
$$

Looking at interaction terms suppressed by the smallest scale, we find

$$
\sim \frac{\left(\partial^{2} \phi\right)^{3}}{M_{P} m^{4}}
$$

corresponding to the decoupling limit:

$$
m \rightarrow 0, \quad M_{P} \rightarrow \infty, \quad \Lambda_{5}, h_{\mu \nu}, A_{\mu}, \phi \text { fixed }
$$

which corresponds to 4 point scattering amplitudes growing with energy like $\sim E^{10}$. However, miraculous cancellations occur and these all vanish with the ghost free potential $[23,47]$. Similarly, interaction coming in at the next smallest scale, given by:

$$
\sim \frac{\left(\partial^{2} \phi\right)^{4}}{\left(M_{P} m^{3}\right)^{2}}, \quad \frac{\partial A\left(\partial^{2} \phi\right)^{2}}{\left(M_{P} m^{3}\right)}
$$

which would correspond to 4 point scattering amplitudes growing with energy like $E^{8}$ also vanish.

The non-vanishing terms with the smallest suppression scale that survive the massless limit are given by:

$$
\sim \frac{h\left(\partial^{2} \phi\right)^{n}}{\left(M_{P} m^{2}\right)^{n-1}}, \quad \frac{(\partial A)^{2}\left(\partial^{2} \phi\right)^{n}}{\left(M_{P} m^{2}\right)^{n}},
$$

and the gauge symmetry reduces to their linear form (A.14). This is found by taking the decoupling limit

$$
m \rightarrow 0, \quad M_{P} \rightarrow \infty, \quad \Lambda_{3}, h_{\mu \nu}, A_{\mu}, \phi \text { fixed }
$$

The remaining interactions give 4 point scattering amplitudes growing like $E^{6}[36]$. In the full non-linear theory, the tensor and scalar modes cannot be fully decoupled from one another, and we get a scalar-tensor theory along with a scalar-vector theory. 
ศ્ต

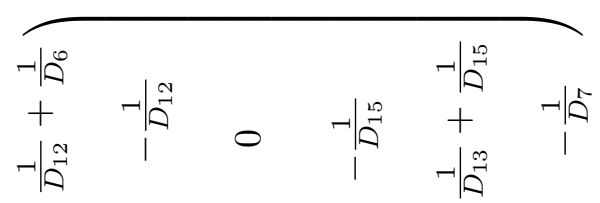

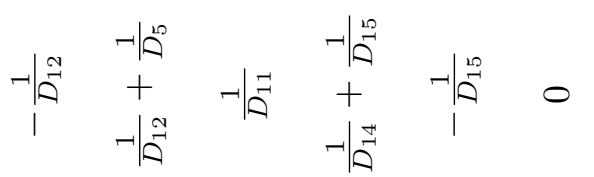

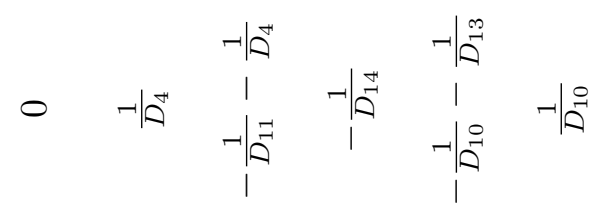

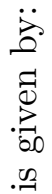

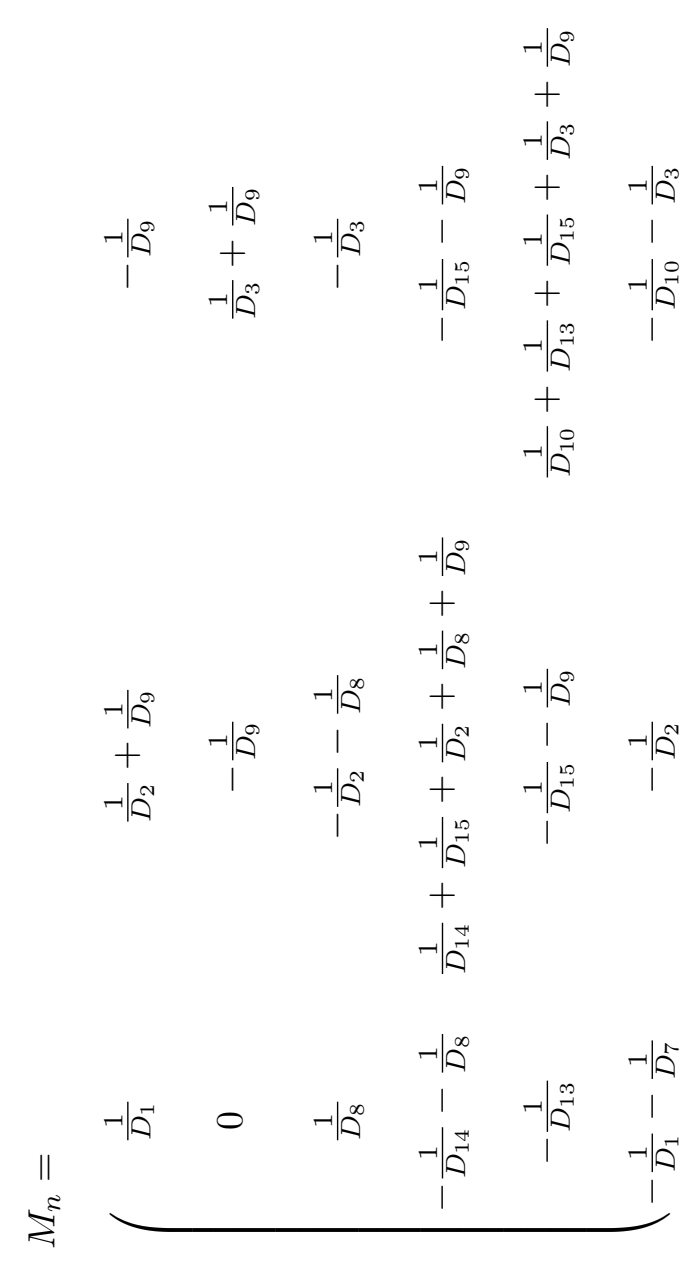

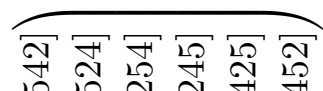

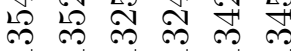

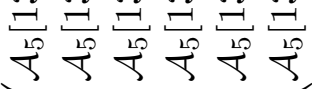

胥

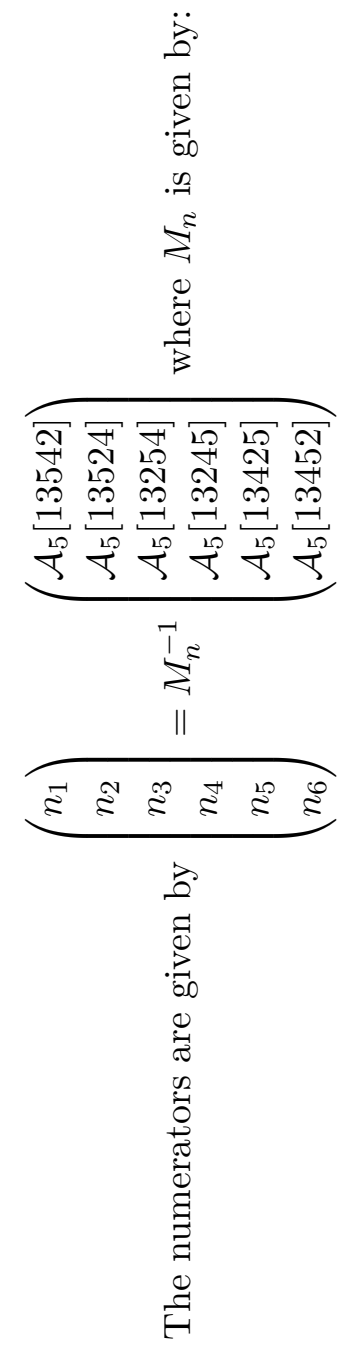

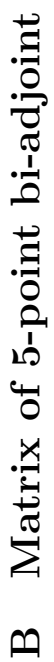

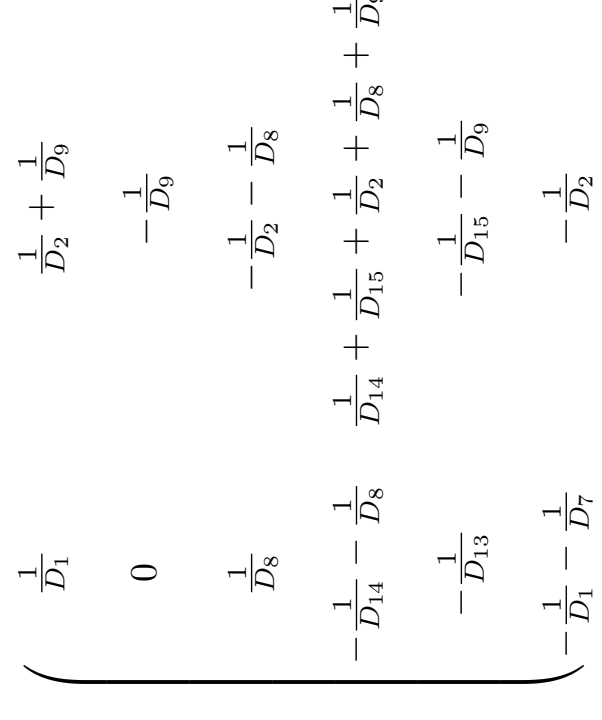




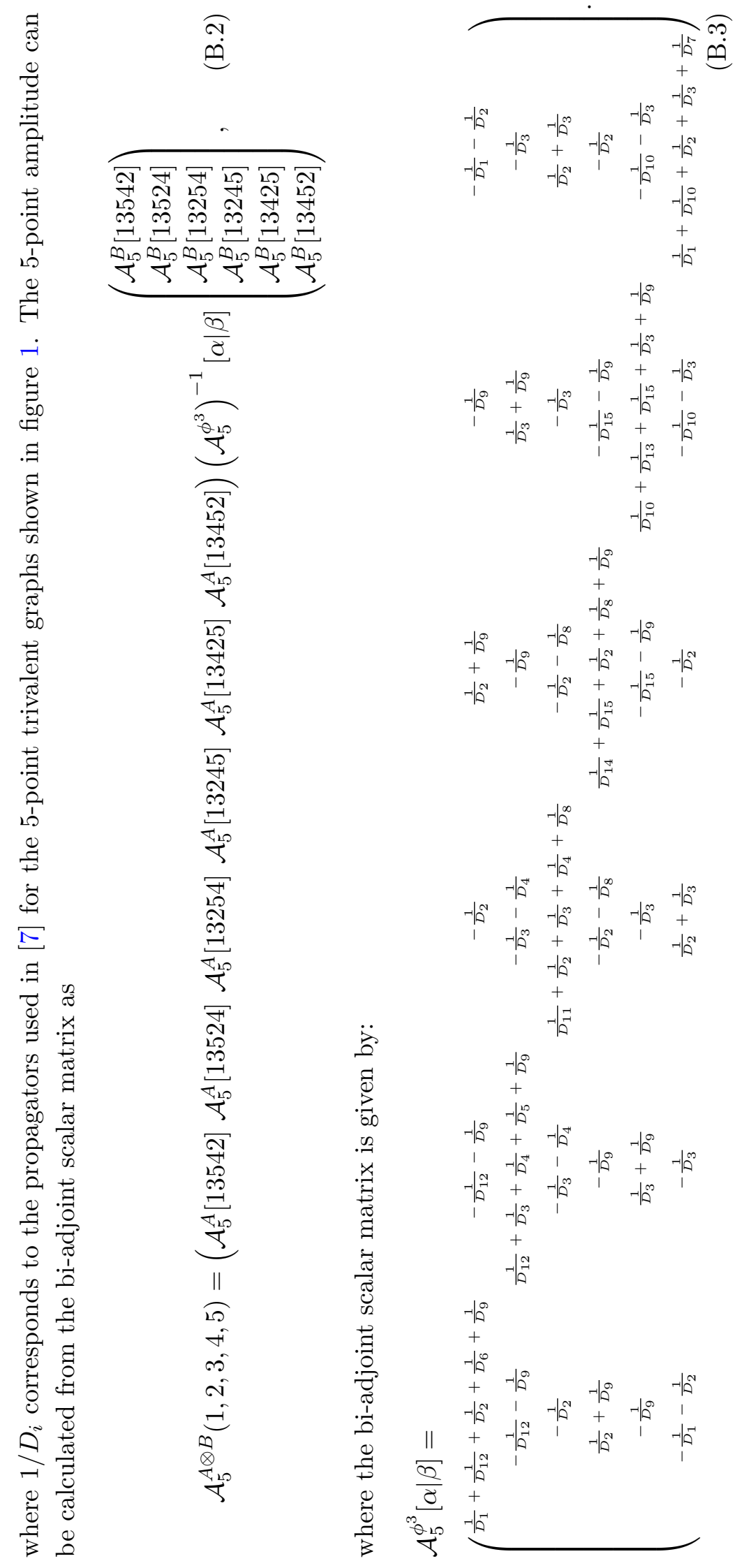




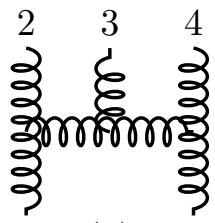

1 (1) 5

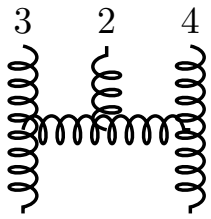

(2) 5

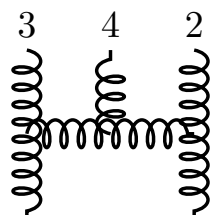

(3) 5

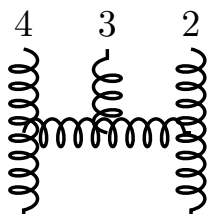

(4) 5

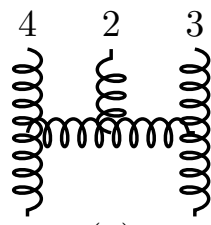

$(5)$

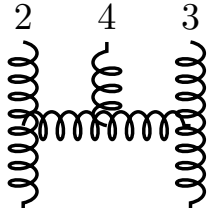

(6) 5

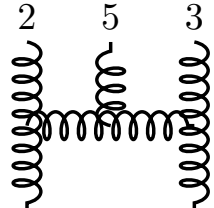

(7)

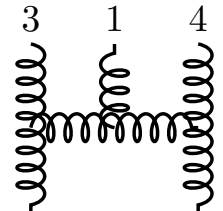

(8) 5

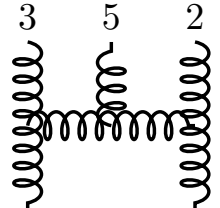

(9) 4

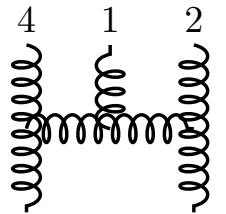

(10) 5
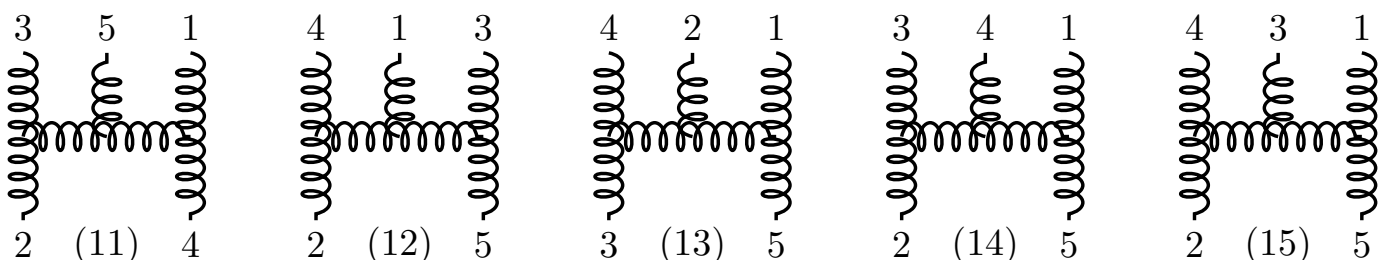

Figure 1. Color-dressed tree-level 5-point amplitude organized using graphs with only cubic vertices.

\section{Factorization on physical poles}

An essential property of amplitudes in local theories is the presence of simple poles when intermediate momenta go on-shell and factorization of the amplitude into products of lower-point amplitudes in the associated residue. In this appendix, we will discuss how these properties are ensured in amplitudes generated by our proposed massive KLT formula (2.16). We will begin by analyzing factorization of (2.16) on two-particle channels, and then extend the result to multi-particle factorization.

We begin by assuming that theories $A$ and $B$ are local and that their amplitudes factorize correctly on two-particle channels,

$$
\begin{aligned}
& \mathcal{A}_{n}^{A}[12, \sigma]=\frac{\mathcal{A}_{3}^{A}\left[12,-P_{12}\right] \mathcal{A}_{n-1}^{A}\left[P_{12}, \sigma\right]}{s_{12}+m^{2}}+\mathcal{O}\left(\left(s_{12}+m^{2}\right)^{0}\right) \\
& \mathcal{A}_{n}^{B}[12, \sigma]=\frac{\mathcal{A}_{3}^{B}\left[12,-P_{12}\right] \mathcal{A}_{n-1}^{B}\left[P_{12}, \sigma\right]}{s_{12}+m^{2}}+\mathcal{O}\left(\left(s_{12}+m^{2}\right)^{0}\right),
\end{aligned}
$$

where there is an implicit sum over states on the right-hand-side.

Next without loss of generality, let us choose to study factorization on the $s_{12}$ pole. We can further assume that we have chosen a DDM basis in which the first $m$ elements have the form $[12 \sigma(3, \cdots, n)]$ where $\sigma$ is a permutation, and no other elements have 1 and 2 adjacent. ${ }^{12}$ Thus only orderings in the first $m$ rows and columns admit poles in $s_{12}$ and

\footnotetext{
${ }^{12}$ For example, the basis $[1 \sigma(2, \cdots, n-1) n]$ where $\sigma$ runs over all $(n-2)$ ! permutations is a DDM basis with $(n-3)$ ! elements of the form $[12 \sigma(3, \cdots, n-1) n]$. One can then choose an ordering of basis elements such that the assumed property is fulfilled.
} 
we can resolve our matrix of bi-adjoint scalar amplitudes into blocks,

$$
\mathcal{A}_{n}^{\phi^{3}}[\alpha \mid \beta]=\left(\begin{array}{cc}
P & Q^{\top} \\
Q & R
\end{array}\right),
$$

where $P, Q$ and $R$ are $m \times m,(n-m) \times m$ and $(n-m) \times(n-m)$ matrices respectively. Since $s_{12}$ poles are not admitted by the last $(n-m)$ orderings, the $P$ matrix contains all the elements with an $s_{12}$ pole and $Q$ and $R$ do not contain any elements with an $s_{12}$ pole. Locality and unitarity of bi-adjoint scalar theory then demands that elements of $Q$ and $R$ will have zero residue on the $s_{12}$ pole, and a given element of $P$ will have the form

$$
\begin{aligned}
\mathcal{A}_{n}^{\phi^{3}}\left[12, \sigma(3, \cdots, n) \mid 12, \sigma^{\prime}(3, \cdots, n)\right]= & \frac{\mathcal{A}_{n-1}^{\phi^{3}}\left[P_{12}, \sigma(3, \cdots, n) \mid P_{12}, \sigma^{\prime}(3, \cdots, n)\right]}{s_{12}+m^{2}} \\
& +\mathcal{O}\left(\left(s_{12}+m^{2}\right)^{0}\right)
\end{aligned}
$$

near the pole. We will now assume that the orderings $\left[P_{12}, \sigma(3, \cdots, n)\right]$ form a DDM basis for $n-1$ particles $\left\{P_{12}, 3,4, \ldots, n\right\} .{ }^{13}$ Thus the blocks are characterized by their behavior as they approach the $s_{12}$ pole,

$$
P=\mathcal{O}\left(\left(s_{12}+m^{2}\right)^{-1}\right), \quad Q=\mathcal{O}\left(\left(s_{12}+m^{2}\right)^{0}\right), \quad R=\mathcal{O}\left(\left(s_{12}+m^{2}\right)^{0}\right) .
$$

Various useful corollaries can be drawn. For example,

$$
P^{-1}=\mathcal{O}\left(\left(s_{12}+m^{2}\right)^{1}\right), \quad R^{-1}=\mathcal{O}\left(\left(s_{12}+m^{2}\right)^{0}\right) .
$$

In fact, (C.3) allows us to be more specific, for $P^{-1}$,

$$
P^{-1}\left[12, \sigma \mid 12, \sigma^{\prime}\right]=\left(s_{12}+m^{2}\right)\left(\mathcal{A}_{n-1}^{\phi^{3}}\right)^{-1}\left[P_{12}, \sigma \mid P_{12}, \sigma^{\prime}\right]+\mathcal{O}\left(\left(s_{12}+m^{2}\right)^{2}\right),
$$

where we will use the shorthand $\sigma=\sigma(3, \cdots, n)$ and $\sigma^{\prime}=\sigma^{\prime}(3, \cdots, n)$ for the rest of the section. Finally, using the geometric series formula for matrices, we get

$$
\left(1-P^{-1} Q^{\top} R^{-1} Q\right)^{-1}=1+\mathcal{O}\left(\left(s_{12}+m^{2}\right)^{1}\right) .
$$

These properties, along with the blockwise inversion formula

$$
\left(\mathcal{A}_{n}^{\phi^{3}}\right)^{-1}[\alpha \mid \beta]=\left(\begin{array}{cc}
P^{-1}\left(1-P^{-1} Q^{\top} R^{-1} Q\right)^{-1} & -P^{-1}\left(1-P^{-1} Q^{\top} R^{-1} Q\right)^{-1} Q^{\top} R^{-1} \\
-R^{-1} Q P^{-1}\left(1-P^{-1} Q^{\top} R^{-1} Q\right)^{-1} & R^{-1}+R^{-1} Q P^{-1}\left(1-P^{-1} Q^{\top} R^{-1} Q\right)^{-1} Q^{\top} R^{-1}
\end{array}\right),
$$

gives

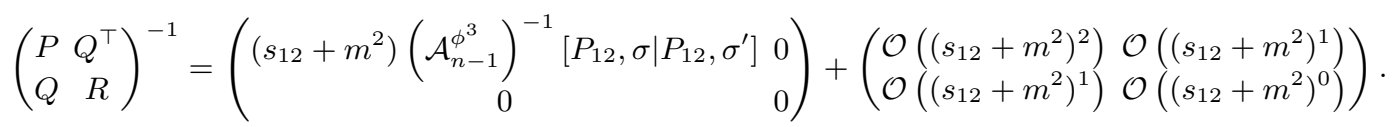

It is straightforward to see that only the elements in the top left block will multiply amplitudes $\mathcal{A}_{3}^{A}[12 \sigma]$ and $\mathcal{A}_{3}^{B}\left[12 \sigma^{\prime}\right]$ and hence only these could develop a pole at $s_{12}$. Thus the suppressed terms on the right-hand-side will not contribute on the factorization channel.

\footnotetext{
${ }^{13}$ Returning to the example DDM basis $[1 \sigma(2, \cdots, n-1) n]$, we see that this condition is satisfied, i.e. $\left[P_{12} \sigma(3, \cdots, n-1) n\right]$ forms a DDM basis.
} 
So in a neighborhood of the $s_{12}$ pole,

$$
\begin{aligned}
\mathcal{A}_{n}^{A \otimes B}= & \sum_{\alpha, \beta} \mathcal{A}_{n}^{A}[\alpha]\left(\mathcal{A}_{n}^{\phi^{3}}\right)^{-1}[\alpha \mid \beta] \mathcal{A}_{n}^{B}[\beta] \\
= & \sum_{\sigma, \sigma^{\prime}} \frac{\mathcal{A}_{3}^{A}\left[12,-P_{12}\right] \mathcal{A}_{n-1}^{A}\left[P_{12}, \sigma\right]\left(\mathcal{A}_{n-1}^{\phi^{3}}\right)^{-1}\left[P_{12}, \sigma \mid P_{12}, \sigma^{\prime}\right] \mathcal{A}_{3}^{B}\left[12,-P_{12}\right] \mathcal{A}_{n-1}^{B}\left[P_{12}, \sigma^{\prime}\right]}{s_{12}+m^{2}} \\
& \quad+\mathcal{O}\left(\left(s_{12}+m^{2}\right)^{0}\right) \\
= & \mathcal{A}_{3}^{A}\left[12,-P_{12}\right] \mathcal{A}_{3}^{B}\left[12,-P_{12}\right] \sum_{\sigma, \sigma^{\prime}} \frac{\mathcal{A}_{n-1}^{A}\left[P_{12}, \sigma\right]\left(\mathcal{A}_{n-1}^{\phi^{3}}\right)^{-1}\left[P_{12}, \sigma \mid P_{12}, \sigma^{\prime}\right] \mathcal{A}_{n-1}^{B}\left[P_{12}, \sigma^{\prime}\right]}{s_{12}+m^{2}} \\
& \quad+\mathcal{O}\left(\left(s_{12}+m^{2}\right)^{0}\right) \quad(\mathrm{C} .10) \\
= & \frac{\mathcal{A}_{3}^{A \otimes B}\left(1,2,-P_{12}\right) \mathcal{A}_{n-1}^{A \otimes B}\left(P_{12}, 3, \ldots, n\right)}{s_{12}+m^{2}}+\mathcal{O}\left(\left(s_{12}+m^{2}\right)^{0}\right),
\end{aligned}
$$

where we have used the fact that for $n=3$, the formula (2.16) takes the simple form,

$$
\mathcal{A}_{3}^{A \otimes B}(1,2,3)=\mathcal{A}_{3}^{A}[123] \mathcal{A}_{3}^{B}[123] .
$$

Thus, on a two-particle channel, an $n$-point amplitude generated by the massive KLT formula factorizes into lower-point amplitudes also generated by (2.16), i.e. these amplitudes factorize into the correct lower-point amplitudes. Since we chose to study the $s_{12}$ pole without loss of generality, this argument demonstrates factorization on all two-particle singularities.

This argument generalizes straightforwardly to multi-particle factorization. Without loss of generality we will consider factorization on the singularity

$$
P^{2}=m^{2}, \quad \text { where } P^{\mu} \equiv p_{1}^{\mu}+p_{2}^{\mu}+\ldots+p_{k-1}^{\mu}+p_{k}^{\mu} .
$$

A double-ordered bi-adjoint scalar amplitude will contain such a singularity only if both its orderings have $\{1,2, \ldots, k\}$ cyclically adjacent. As above, we choose a DDM basis for the $n$-point amplitudes in which the minimal number of amplitudes with a $P^{2}$ factorization singularity appear. A natural choice is

$$
\left\{\mathcal{A}_{n}^{\phi^{3}}[1, \alpha, n \mid 1, \beta, n]: \alpha, \beta \in \mathcal{P}(2,3, \ldots, n-1)\right\} .
$$

The subset of these amplitudes which have a $P^{2}=m^{2}$ singularity have the form

$$
\left\{\mathcal{A}_{n}^{\phi^{3}}\left[1, \sigma, \rho, n \mid 1, \sigma^{\prime}, \rho^{\prime}, n\right]: \sigma, \sigma^{\prime} \in \mathcal{P}(2,3, \ldots, k-1, k), \rho, \rho^{\prime} \in \mathcal{P}(k+1, k+2, \ldots, n-1, n)\right\} .
$$

Near the singularity such amplitudes have the form

$\mathcal{A}_{n}^{\phi^{3}}\left[1, \sigma, \rho, n \mid 1, \sigma^{\prime}, \rho^{\prime}, n\right]=\frac{\mathcal{A}_{k+1}^{\phi^{3}}\left[1, \sigma,-P \mid 1, \sigma^{\prime},-P\right] \mathcal{A}_{k+1}^{\phi^{3}}\left[P, \rho, n \mid P, \rho^{\prime}, n\right]}{P^{2}+m^{2}}+\mathcal{O}\left(\left(P^{2}+m^{2}\right)^{0}\right)$. 
Placing all such amplitudes in the top-left-hand corner of the matrix of biadjoint-scalar amplitudes, we obtain the same result as in section 2.2 , that only amplitudes of this form are important on the factorization channel when using the block decomposition inverse formula (C.8). Here the associated subspaces are indexed by a pair of orderings $(\sigma, \rho)$ on the left and $\left(\sigma^{\prime}, \rho^{\prime}\right)$ on the right. The required inverse is then given by

$$
\begin{aligned}
& \left(\mathcal{A}_{n}^{\phi^{3}}\right)^{-1}\left[1, \sigma, \rho, n \mid 1, \sigma^{\prime}, \rho^{\prime}, n\right] \\
& =\left(P^{2}+m^{2}\right)\left(\mathcal{A}_{k+1}^{\phi^{3}}\right)^{-1}\left[1, \sigma,-P \mid 1, \sigma^{\prime},-P\right]\left(\mathcal{A}_{k+1}^{\phi^{3}}\right)^{-1}\left[P, \rho, n \mid P, \rho^{\prime}, n\right]+\mathcal{O}\left(\left(P^{2}+m^{2}\right)^{2}\right) .
\end{aligned}
$$

This is an application of a general result for the so-called Kronecker product of matrices

$$
(P \otimes Q)^{-1}=P^{-1} \otimes Q^{-1} .
$$

Verifying that this is true is trivial in component form. We label the components as $P_{i k}$ and $Q_{j l}$, the Kronecker product is then defined component-wise as $(P \otimes Q)_{i j k l} \equiv P_{i k} Q_{j l}$. The right-inverse is defined to satisfy

$$
\sum_{m, n}(P \otimes Q)_{i j m n}(P \otimes Q)_{m n k l}^{-1}=\delta_{i k} \delta_{j l} .
$$

It is straightforward to see that this is satisfied by matrices of the form

$$
(P \otimes Q)_{m n k l}^{-1}=\left(P^{-1}\right)_{m k}\left(Q^{-1}\right)_{n l},
$$

and similarly for the left-inverse. Using this result, on the neighborhood of the $P^{2}=m^{2}$ pole,

$$
\begin{aligned}
\mathcal{A}_{n}^{A \otimes B}(1,2, \ldots, n) & \sum_{\alpha, \beta} \mathcal{A}_{n}^{A}[\alpha]\left(\mathcal{A}_{n}^{\phi^{3}}\right)^{-1}[\alpha \mid \beta] \mathcal{A}_{n}^{B}[\beta] \\
= & \sum_{\sigma, \sigma^{\prime}} \sum_{\rho \rho^{\prime}} \frac{1}{\left(P^{2}+m^{2}\right)^{2}}\left(\mathcal{A}_{k+1}^{A}[1, \sigma,-P] \mathcal{A}_{n-k+1}^{A}[P, \rho, n] \times\left(\mathcal{A}_{n}^{\phi^{3}}\right)^{-1}\left[1, \sigma, \rho, n \mid 1, \sigma^{\prime}, \rho^{\prime}, n\right]\right. \\
& \left.\times \mathcal{A}_{k+1}^{B}\left[1, \sigma^{\prime},-P\right] \mathcal{A}_{n-k+1}^{B}\left[P, \rho^{\prime}, n\right]\right)+\mathcal{O}\left(\left(P^{2}+m^{2}\right)^{0}\right) \\
= & \sum_{\sigma, \sigma^{\prime}} \sum_{\rho \rho^{\prime}} \frac{1}{P^{2}+m^{2}}\left(\mathcal{A}_{k+1}^{A}[1, \sigma,-P] \mathcal{A}_{n-k+1}^{A}[P, \rho, n]\left(\mathcal{A}_{k+1}^{\phi^{3}}\right)^{-1}\left[1, \sigma,-P \mid 1, \sigma^{\prime},-P\right]\right. \\
& \left.\times\left(\mathcal{A}_{n-k+1}^{\phi^{3}}\right)^{-1}\left[P, \rho, n \mid P, \rho^{\prime}, n\right] \mathcal{A}_{k+1}^{B}\left[1, \sigma^{\prime},-P\right] \mathcal{A}_{n-k+1}^{B}\left[P, \rho^{\prime}, n\right]\right)+\mathcal{O}\left(\left(P^{2}+m^{2}\right)^{0}\right) \\
= & \frac{1}{P^{2}+m^{2}}\left(\sum_{\sigma, \sigma^{\prime}} \mathcal{A}_{k+1}^{A}[1, \sigma,-P]\left(\mathcal{A}_{k+1}^{\phi^{3}}\right)^{-1}\left[1, \sigma,-P \mid 1, \sigma^{\prime},-P\right] \mathcal{A}_{k+1}^{B}\left[1, \sigma^{\prime},-P\right]\right) \\
& \times\left(\sum_{\rho \rho^{\prime}} \mathcal{A}_{n-k+1}^{A}[P, \rho, n]\left(\mathcal{A}_{n-k+1}^{\phi^{3}}\right)^{-1}\left[P, \rho, n \mid P, \rho^{\prime}, n\right] \mathcal{A}_{n-k+1}^{B}\left[P, \rho^{\prime}, n\right]\right)+\mathcal{O}\left(\left(P^{2}+m^{2}\right)^{0}\right) \\
= & \frac{\mathcal{A}_{k+1}^{A \otimes B}(1,2, \ldots, k,-P) \mathcal{A}_{n-k+1}^{A \otimes B}(P, k+1, \ldots, n)}{P^{2}+m^{2}}+\mathcal{O}\left(\left(P^{2}+m^{2}\right)^{0}\right) .
\end{aligned}
$$


So we find that the massive KLT formula generates expressions which factor correctly on all singularities.

\section{Feynman rules for massive Yang-Mills}

At low multiplicity it is efficient to calculate the scattering amplitudes of massive YangMills (3.6) using Feynman rules. The vertex functions are identical to those of standard non-Abelian gauge theory:

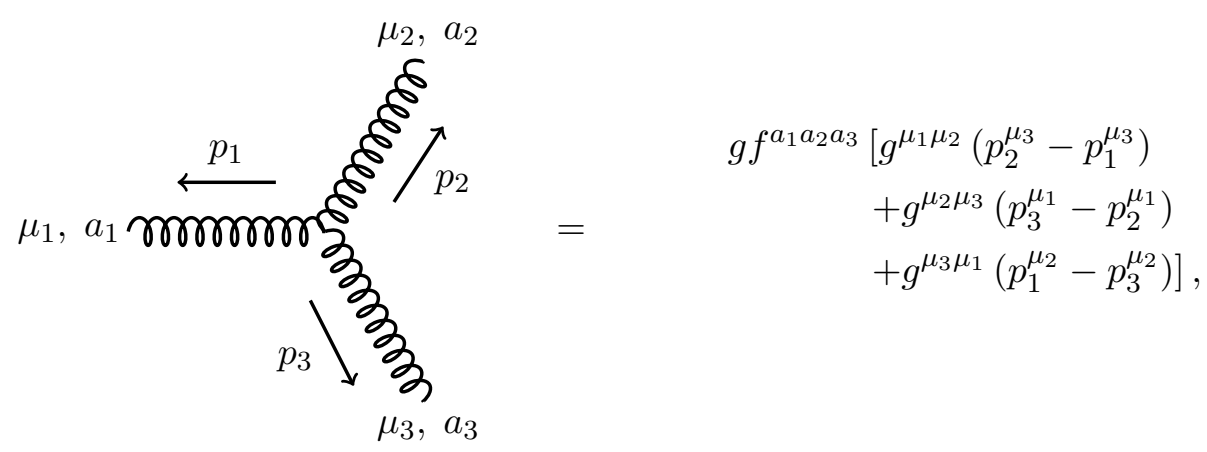

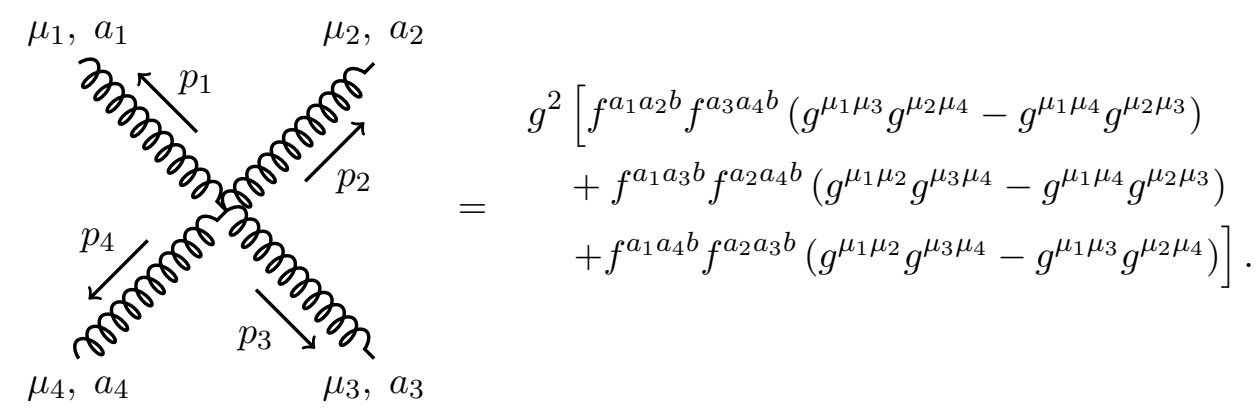

Meanwhile the propagator is modified to take the Proca form:

$$
\stackrel{p}{\stackrel{p}{\longrightarrow}} \nu, b=\frac{\delta^{a b}}{p^{2}+m^{2}}\left(g_{\mu \nu}+\frac{p_{\mu} p_{\nu}}{m^{2}}\right) \text {. }
$$

\section{E 4-point graviton-dilaton amplitudes from double-copy}

The amplitudes given by the double-copy of massive Yang-Mills are given here:

$$
\begin{aligned}
\mathcal{M}_{4}^{h h h h}= & -\frac{1}{4 M_{p}^{2}}\left(\frac { 1 } { m ^ { 2 } - 2 p _ { 1 2 } } \left(-z_{14} z_{23} m^{2}+z_{13} z_{24} m^{2}+2 z_{12} z_{34} m^{2}+2 p_{12} z_{14} z_{23}\right.\right. \\
& -2 p_{12} z_{13} z_{24}-2 p_{12} z_{12} z_{34}-4 p_{13} z_{12} z_{34}+4 z_{34} z p_{13} z p_{21}-4 z_{34} z p_{12} z p_{23} \\
& +4 z_{24} z p_{12} z p_{31}-4 z_{14} z p_{21} z p_{31}+4 z_{24} z p_{12} z p_{32}-4 z_{14} z p_{21} z p_{32}-4 z_{23} z p_{12} z p_{41} \\
& \left.+4 z_{13} z p_{21} z p_{41}+4 z_{12} z p_{32} z p_{41}-4 z_{23} z p_{12} z p_{42}+4 z_{13} z p_{21} z p_{42}-4 z_{12} z p_{31} z p_{42}\right)^{2} \\
& +(2 \leftrightarrow 3)+(2 \leftrightarrow 4)) .
\end{aligned}
$$




$$
\begin{aligned}
\mathcal{M}_{4}^{\phi \phi \phi \phi}= & \frac{1}{M_{p}^{2}}\left(-\frac{p_{13}^{2}\left(75 m^{2} p_{12}+34 p_{12}^{2}+116 m^{4}\right)}{72 m^{4}\left(m^{2}-2 p_{12}\right)}\right. \\
& +\frac{3}{64}\left(-24 m^{2} p_{12}+48 p_{12}^{2}+115 m^{4}\right)\left(\frac{1}{2 p_{14}+m^{2}}+\frac{1}{2 p_{13}+m^{2}}\right) \\
& +\frac{p_{13}\left(-41 m^{4} p_{12}-41 m^{2} p_{12}^{2}-34 p_{12}^{3}+116 m^{6}\right)}{72 m^{4}\left(m^{2}-2 p_{12}\right)} \\
& \left.-\frac{-4751 m^{4} p_{12}+744 m^{2} p_{12}^{2}+368 p_{12}^{3}+3696 m^{6}}{288 m^{2}\left(m^{2}-2 p_{12}\right)}\right) \\
\mathcal{M}_{4}^{h \phi \phi \phi}= & \frac{1}{6 \sqrt{3} m^{4} M_{p}^{2}\left(m^{2}-2 p_{12}\right)\left(m^{2}-2 p_{13}\right)\left(m^{2}-2\left(p_{14}\right)\right)}\left(m ^ { 1 0 } \left(19 z p_{12}^{2}-43 z p_{13} z p_{12}\right.\right. \\
& \left.+19 z p_{13}^{2}\right)-m^{8}\left(p_{13}\left(136 z p_{12}^{2}-53 z p_{13} z p_{12}+76 z p_{13}^{2}\right)+p_{12}\left(76 z p_{12}^{2}-53 z p_{13} z p_{12}\right.\right. \\
& \left.\left.+136 z p_{13}^{2}\right)\right)+m^{6}\left(p_{12}^{2}\left(76 z p_{12}^{2}+61 z p_{13} z p_{12}+195 z p_{13}^{2}\right)+3 p_{13} p_{12}\left(39 z p_{12}^{2}\right.\right. \\
& \left.\left.-53 z p_{13} z p_{12}+39 z p_{13}^{2}\right)+p_{13}^{2}\left(195 z p_{12}^{2}+61 z p_{13} z p_{12}+76 z p_{13}^{2}\right)\right) \\
& +m^{4}\left(p_{12}^{3} z p_{13}\left(10 z p_{12}+z p_{13}\right)+p_{13} p_{12}^{2}\left(-41 z p_{12}^{2}+10 z p_{13} z p_{12}-37 z p_{13}^{2}\right)\right. \\
& \left.+p_{13}^{2} p_{12}\left(-37 z p_{12}^{2}+10 z p_{13} z p_{12}-41 z p_{13}^{2}\right)+p_{13}^{3} z p_{12}\left(z p_{12}+10 z p_{13}\right)\right) \\
& +2 m^{2}\left(p_{12}^{4} z p_{13}^{2}-12 p_{13} p_{12}^{3} z p_{12} z p_{13}-2 p_{13}^{2} p_{12}^{2}\left(z p_{12}^{2}+13 z p_{13} z p_{12}+z p_{13}^{2}\right)\right. \\
& \left.\left.\left.-12 p_{13}^{3} p_{12} z p_{12} z p_{13}+p_{13}^{4} z p_{12}^{2}\right)-4 p_{12} p_{13}\left(p_{12}+p_{13}\right)\left(p_{13} z p_{12}-p_{12} z p_{13}\right)^{2}\right)\right) .
\end{aligned}
$$

$$
\begin{aligned}
& \mathcal{M}_{4}^{h h \phi \phi}=\frac{1}{6 m^{2} M_{p}^{2}\left(m^{2}-2 p_{12}\right)\left(m^{2}-2 p_{13}\right)\left(m^{2}-2 p_{14}\right)}\left(19 z_{12}^{2} m^{10}\right. \\
& -z_{12}\left(62 p_{12} z_{12}+92 p_{13} z_{12}+z p_{12} z p_{21}-35 z p_{13} z p_{21}+17 z p_{12} z p_{23}-18 z p_{13} z p_{23}\right) m^{8} \\
& +\left(42 p_{12}^{2} z_{12}^{2}+156 p_{13}^{2} z_{12}^{2}+4 p_{13}\left(z p_{12}\left(z p_{21}+21 z p_{23}\right)-3 z p_{13}\left(13 z p_{21}+6 z p_{23}\right)\right) z_{12}\right. \\
& +p_{12}\left(180 p_{13} z_{12}+z p_{12}\left(4 z p_{21}+31 z p_{23}\right)-z p_{13}\left(121 z p_{21}+90 z p_{23}\right)\right) z_{12}+34 z p_{13}^{2} z p_{21}^{2} \\
& \left.+z p_{12}^{2} z p_{23}^{2}-33 z p_{12} z p_{13} z p_{23}^{2}+33 z p_{13}^{2} z p_{21} z p_{23}-35 z p_{12} z p_{13} z p_{21} z p_{23}\right) m^{6} \\
& +\left(13 z_{12}^{2} p_{12}^{3}-4 z_{12}\left(14 p_{13} z_{12}+z p_{12}\left(z p_{21}-2 z p_{23}\right)-z p_{13}\left(25 z p_{21}+27 z p_{23}\right)\right) p_{12}^{2}\right. \\
& -\left(200 p_{13}^{2} z_{12}^{2}+12 p_{13}\left(z p_{12}\left(z p_{21}+10 z p_{23}\right)-2 z p_{13}\left(14 z p_{21}+9 z p_{23}\right)\right) z_{12}+z p_{12}^{2} z p_{23}^{2}\right. \\
& \left.-2 z p_{12} z p_{13} z p_{23}\left(34 z p_{21}+33 z p_{23}\right)+z p_{13}^{2}\left(100 z p_{21}^{2}+132 z p_{23} z p_{21}+33 z p_{23}^{2}\right)\right) p_{12} \\
& -p_{13}\left(128 p_{13}^{2} z_{12}^{2}+4 p_{13}\left(z p_{12}\left(z p_{21}+42 z p_{23}\right)-6 z p_{13}\left(10 z p_{21}+3 z p_{23}\right)\right) z_{12}\right. \\
& \left.+37 z p_{12}^{2} z p_{23}^{2}-2 z p_{12} z p_{13} z p_{23}\left(70 z p_{21}+33 z p_{23}\right)+z p_{13}^{2} z p_{21}\left(103 z p_{21}+66 z p_{23}\right)\right) m^{4} \\
& -2\left(z_{12}^{2} p_{12}^{4}+2 z_{12}\left(9 p_{13} z_{12}-z p_{13} z p_{21}+z p_{12} z p_{23}\right) p_{12}^{3}+\left(-\left(32 z p_{21}^{2}+66 z p_{23} z p_{21}\right.\right.\right. \\
& \left.+33 z p_{23}^{2}\right) z p_{13}^{2}-2 z p_{12} z p_{21} z p_{23} z p_{13}+z p_{12}^{2} z p_{23}^{2}+2 p_{13} z_{12}\left(z p_{12}\left(7 z p_{23}-2 z p_{21}\right)\right. \\
& \left.\left.+z p_{13}\left(29 z p_{21}+36 z p_{23}\right)\right)\right) p_{12}^{2}-2 p_{13}\left(24 p_{13}^{2} z_{12}^{2}+p_{13}\left(z p_{12}\left(2 z p_{21}+27 z p_{23}\right)\right.\right. \\
& \left.\left.-9 z p_{13}\left(7 z p_{21}+4 z p_{23}\right)\right) z_{12}+33 z p_{13}\left(z p_{21}+z p_{23}\right)\left(z p_{13} z p_{21}-z p_{12} z p_{23}\right)\right) p_{12} \\
& \left.-p_{13}^{2}\left(32 p_{13}^{2} z_{12}^{2}-68 p_{13}\left(z p_{13} z p_{21}-z p_{12} z p_{23}\right) z_{12}+35\left(z p_{13} z p_{21}-z p_{12} z p_{23}\right)^{2}\right)\right) m^{2} \\
& \left.\left.+4 p_{12} p_{13}\left(p_{12}+p_{13}\right)\left(p_{12} z_{12}+2 p_{13} z_{12}-z p_{13} z p_{21}+z p_{12} z p_{23}\right)^{2}\right)\right) \text {. }
\end{aligned}
$$




$$
\begin{aligned}
& \mathcal{M}_{4}^{h h h \phi}=\frac{-1}{2 \sqrt{3} M_{p}^{2}\left(m^{2}-2 p_{12}\right)\left(m^{2}-2 p_{13}\right)\left(m^{2}-2\left(p_{14}\right)\right.}\left(z_{12} z_{13} z_{23} m^{8}-\left(-14 z p_{31}^{2} z_{12}^{2}\right.\right. \\
& -2 z p_{32}^{2} z_{12}^{2}-8 z p_{31} z p_{32} z_{12}^{2}+4 p_{12} z_{13} z_{23} z_{12}+4 p_{13} z_{13} z_{23} z_{12}-7 z_{23} z p_{12} z p_{31} z_{12} \\
& +13 z_{23} z p_{13} z p_{31} z_{12}+13 z_{13} z p_{21} z p_{31} z_{12}-7 z_{13} z p_{23} z p_{31} z_{12}+z_{23} z p_{12} z p_{32} z_{12} \\
& +5 z_{23} z p_{13} z p_{32} z_{12}-7 z_{13} z p_{21} z p_{32} z_{12}-11 z_{13} z p_{23} z p_{32} z_{12}+z_{23}^{2} z p_{12}^{2}+z_{23}^{2} z p_{13}^{2} \\
& -14 z_{13}^{2} z p_{21}^{2}-2 z_{13}^{2} z p_{23}^{2}+10 z_{23}^{2} z p_{12} z p_{13}+13 z_{13} z_{23} z p_{12} z p_{21}-7 z_{13} z_{23} z p_{13} z p_{21} \\
& \left.+5 z_{13} z_{23} z p_{12} z p_{23}+z_{13} z_{23} z p_{13} z p_{23}-8 z_{13}^{2} z p_{21} z p_{23}\right) m^{6}+\left(4 z_{12} z_{13} z_{23} p_{12}^{2}\right. \\
& +\left(-41 z p_{31}^{2} z_{12}^{2}-5 z p_{32}^{2} z_{12}^{2}-14 z p_{31} z p_{32} z_{12}^{2}+12 p_{13} z_{13} z_{23} z_{12}+40 z_{13} z p_{21} z p_{31} z_{12}\right. \\
& -28 z_{13} z p_{23} z p_{31} z_{12}-16 z_{13} z p_{21} z p_{32} z_{12}-20 z_{13} z p_{23} z p_{32} z_{12}-44 z_{13}^{2} z p_{21}^{2}-20 z_{13}^{2} z p_{23}^{2} \\
& +z_{23}^{2}\left(z p_{12}^{2}+22 z p_{13} z p_{12}-11 z p_{13}^{2}\right)-32 z_{13}^{2} z p_{21} z p_{23}+4 z_{23}\left(z _ { 1 3 } \left(z p_{12}\left(7 z p_{21}+2 z p_{23}\right)\right.\right. \\
& \left.\left.\left.+z p_{13}\left(4 z p_{23}-7 z p_{21}\right)\right)+z_{12}\left(z p_{12}\left(z p_{32}-4 z p_{31}\right)+z p_{13}\left(13 z p_{31}+2 z p_{32}\right)\right)\right)\right) p_{12} \\
& +p_{13}\left(-44 z p_{31}^{2} z_{12}^{2}-20 z p_{32}^{2} z_{12}^{2}-32 z p_{31} z p_{32} z_{12}^{2}+4 p_{13} z_{13} z_{23} z_{12}+40 z_{13} z p_{21} z p_{31} z_{12}\right. \\
& -16 z_{13} z p_{23} z p_{31} z_{12}-28 z_{13} z p_{21} z p_{32} z_{12}-20 z_{13} z p_{23} z p_{32} z_{12}-41 z_{13}^{2} z p_{21}^{2}-5 z_{13}^{2} z p_{23}^{2} \\
& +z_{23}^{2}\left(-11 z p_{12}^{2}+22 z p_{13} z p_{12}+z p_{13}^{2}\right)-14 z_{13}^{2} z p_{21} z p_{23}+4 z_{23}\left(z _ { 1 3 } \left(z p_{13}\left(z p_{23}-4 z p_{21}\right)\right.\right. \\
& \left.\left.\left.\left.+z p_{12}\left(13 z p_{21}+2 z p_{23}\right)\right)+z_{12}\left(z p_{13}\left(7 z p_{31}+2 z p_{32}\right)+z p_{12}\left(4 z p_{32}-7 z p_{31}\right)\right)\right)\right)\right) m^{4} \\
& +2\left(\left(13 z p_{31}^{2} z_{12}^{2}+z p_{32}^{2} z_{12}^{2}-2 z p_{31} z p_{32} z_{12}^{2}-4 p_{13} z_{13} z_{23} z_{12}-14 z_{13} z p_{21} z p_{31} z_{12}\right.\right. \\
& +14 z_{13} z p_{23} z p_{31} z_{12}+2 z_{13} z p_{21} z p_{32} z_{12}-2 z_{13} z p_{23} z p_{32} z_{12}+16 z_{13}^{2} z p_{21}^{2}+16 z_{13}^{2} z p_{23}^{2} \\
& -2 z_{23}\left(z_{13}\left(z p_{12}-7 z p_{13}\right)\left(z p_{21}-z p_{23}\right)+z_{12} z p_{13}\left(13 z p_{31}-z p_{32}\right)\right. \\
& \left.\left.+z_{12} z p_{12}\left(z p_{32}-z p_{31}\right)\right)+z_{23}^{2}\left(z p_{12}^{2}-2 z p_{13} z p_{12}+13 z p_{13}^{2}\right)+16 z_{13}^{2} z p_{21} z p_{23}\right) p_{12}^{2} \\
& -2 p_{13}\left(2 p_{13} z_{12} z_{13} z_{23}-3\left(\left(5 z p_{31}^{2}+2 z p_{32} z p_{31}+z p_{32}^{2}\right) z_{12}^{2}+z_{13}\left(3 z p_{23}\left(z p_{31}+z p_{32}\right)\right.\right.\right. \\
& \left.+z p_{21}\left(3 z p_{32}-5 z p_{31}\right)\right) z_{12}-4 z_{23}^{2} z p_{12} z p_{13}+z_{13}^{2}\left(5 z p_{21}^{2}+2 z p_{23} z p_{21}+z p_{23}^{2}\right) \\
& -z_{23}\left(z_{13}\left(z p_{13}\left(z p_{23}-3 z p_{21}\right)+z p_{12}\left(5 z p_{21}+z p_{23}\right)\right)+z_{12}\left(z p_{12}\left(z p_{32}-3 z p_{31}\right)\right.\right. \\
& \left.\left.\left.\left.+z p_{13}\left(5 z p_{31}+z p_{32}\right)\right)\right)\right)\right) p_{12}+p_{13}^{2}\left(16\left(z p_{31}^{2}+z p_{32} z p_{31}+z p_{32}^{2}\right) z_{12}^{2}\right. \\
& -2 z_{13}\left(7 z p_{21}-z p_{23}\right)\left(z p_{31}-z p_{32}\right) z_{12}+z_{23}^{2}\left(13 z p_{12}^{2}-2 z p_{13} z p_{12}+z p_{13}^{2}\right) \\
& +z_{13}^{2}\left(13 z p_{21}^{2}-2 z p_{23} z p_{21}+z p_{23}^{2}\right)-2 z_{23}\left(z _ { 1 3 } \left(z p_{12}\left(13 z p_{21}-z p_{23}\right)\right.\right. \\
& \left.\left.\left.\left.+z p_{13}\left(z p_{23}-z p_{21}\right)\right)-z_{12}\left(7 z p_{12}-z p_{13}\right)\left(z p_{31}-z p_{32}\right)\right)\right)\right) m^{2} \\
& \left.-4 p_{12} p_{13}\left(p_{12}+p_{13}\right)\left(z_{23}\left(z p_{12}-z p_{13}\right)+z_{13}\left(z p_{23}-z p_{21}\right)+z_{12}\left(z p_{31}-z p_{32}\right)\right)^{2}\right) \text {. }
\end{aligned}
$$

\section{F BCJ relations as null vectors}

The BCJ relations can be obtained as null space relations of the matrix of bi-adjoint scalar amplitudes. To show this, one must first notice a remarkable property about these amplitudes. Just as in the massless case, bi-adjoint scalar theory acts as an identity for the massive double-copy, ${ }^{14}$ i.e.

$$
A \otimes \mathrm{BS}=A .
$$

\footnotetext{
${ }^{14}$ It is an interesting fact that this is true whether or not the spectral conditions hold.
} 
To express this in matrix notation, let us first choose an $(n-2)$ ! DDM basis. From this, we choose BCJ-independent $(n-3)$ ! sub-bases $\alpha, \beta$ and $\gamma$ and use the KLT formula,

$$
\mathcal{A}^{\phi^{3}}[\alpha \mid \beta] \mathcal{A}^{\phi^{3}}[\beta \mid \gamma]^{-1} \overrightarrow{\mathcal{A}}^{A}[\gamma]=\overrightarrow{\mathcal{A}}^{A}[\alpha] .
$$

The BCJ relations are consistency conditions that make the KLT formula basis-independent. For example, consider another $(n-3)$ ! sub-basis $\tilde{\gamma}$. We can then express a BCJ relation as

$$
\mathcal{A}^{\phi^{3}}[\alpha \mid \beta] \mathcal{A}^{\phi^{3}}[\beta \mid \gamma]^{-1} \overrightarrow{\mathcal{A}}^{A}[\gamma]=\mathcal{A}^{\phi^{3}}[\alpha \mid \beta] \mathcal{A}^{\phi^{3}}[\beta \mid \tilde{\gamma}]^{-1} \overrightarrow{\mathcal{A}}^{A}[\tilde{\gamma}] .
$$

We now embed these matrices in our original $(n-2)$ ! DDM basis. The matrix $\mathcal{A}^{\phi^{3}}[\alpha \mid \beta]$ is padded with the remaining bi-adjoint scalar amplitudes, while we pad the vector

$$
\left(\mathcal{A}^{\phi^{3}}[\beta \mid \gamma]^{-1} \overrightarrow{\mathcal{A}}^{A}[\gamma]-\mathcal{A}^{\phi^{3}}[\beta \mid \tilde{\gamma}]^{-1} \overrightarrow{\mathcal{A}}^{A}[\tilde{\gamma}]\right)
$$

with zeros. This gives us the following null vector equation,

$$
\mathcal{A}^{\phi^{3}}[\alpha \mid \beta]\left(\mathcal{A}^{\phi^{3}}[\beta \mid \gamma]^{-1} \overrightarrow{\mathcal{A}}^{A}[\gamma]-\mathcal{A}^{\phi^{3}}[\beta \mid \tilde{\gamma}]^{-1} \overrightarrow{\mathcal{A}}^{A}[\tilde{\gamma}]\right)=0 .
$$

To connect this to the BCJ relations of theory $A$, we consider a double-copy of $A$ with itself,

$$
A \otimes A=B
$$

Choosing the same sub-bases as previously, we can rewrite the KLT formula,

$$
\overrightarrow{\mathcal{A}}^{A}[\beta]^{T} \mathcal{A}^{\phi^{3}}[\beta \mid \gamma]^{-1} \overrightarrow{\mathcal{A}}^{A}[\gamma]=\overrightarrow{\mathcal{A}}^{B} .
$$

Again the BCJ relations are given by demanding basis-independence of this formula,

$$
\begin{aligned}
& \overrightarrow{\mathcal{A}}^{A}[\beta]^{T} \mathcal{A}^{\phi^{3}}[\beta \mid \gamma]^{-1} \overrightarrow{\mathcal{A}}^{A}[\gamma]=\overrightarrow{\mathcal{A}}^{A}[\beta]^{T} \mathcal{A}^{\phi^{3}}[\beta \mid \tilde{\gamma}]^{-1} \overrightarrow{\mathcal{A}}^{A}[\tilde{\gamma}] \\
\Rightarrow & \overrightarrow{\mathcal{A}}^{A}[\beta]^{T}\left(\mathcal{A}^{\phi^{3}}[\beta \mid \gamma]^{-1} \overrightarrow{\mathcal{A}}^{A}[\gamma]-\mathcal{A}^{\phi^{3}}[\beta \mid \tilde{\gamma}]^{-1} \overrightarrow{\mathcal{A}}^{A}[\tilde{\gamma}]\right)=0 .
\end{aligned}
$$

We recognize this vector as being a null vector of $\mathcal{A}^{\phi^{3}}[\alpha \mid \beta]$. Indeed this equation must hold for all choices of $\gamma$ and $\tilde{\gamma}$. At 4- and 5-point, we observe that different choices of $\gamma$ and $\tilde{\gamma}$ span the null space of $\mathcal{A}^{\phi^{3}}[\alpha \mid \beta]$, allowing us to generalize this equation to,

$$
\overrightarrow{\mathcal{A}}^{A}[\beta] \cdot \vec{n}=0,
$$

where the vector $\vec{n}$ is any null vector of the matrix of bi-adjoint scalar amplitudes.

Thus (F.9) is an equivalent representation of the BCJ relations. Since the number of null vectors of $\mathcal{A}^{\phi^{3}}[\alpha \mid \beta]$ is exactly the number of independent BCJ relations, we expect this equivalence to continue to any $n$-point.

Open Access. This article is distributed under the terms of the Creative Commons Attribution License (CC-BY 4.0), which permits any use, distribution and reproduction in any medium, provided the original author(s) and source are credited. 


\section{References}

[1] H. Kawai, D.C. Lewellen and S.H.H. Tye, A Relation Between Tree Amplitudes of Closed and Open Strings, Nucl. Phys. B 269 (1986) 1 [INSPIRE].

[2] H. Johansson and A. Ochirov, Pure Gravities via Color-Kinematics Duality for Fundamental Matter, JHEP 11 (2015) 046 [arXiv:1407.4772] [INSPIRE].

[3] H. Johansson and A. Ochirov, Color-Kinematics Duality for QCD Amplitudes, JHEP 01 (2016) 170 [arXiv: 1507.00332] [inSPIRE].

[4] F. Cachazo, S. He and E.Y. Yuan, Scattering Equations and Matrices: From Einstein To Yang-Mills, DBI and NLSM, JHEP 07 (2015) 149 [arXiv:1412.3479] [INSPIRE].

[5] Z. Bern, J.J.M. Carrasco and H. Johansson, Perturbative Quantum Gravity as a Double Copy of Gauge Theory, Phys. Rev. Lett. 105 (2010) 061602 [arXiv:1004.0476] [INSPIRE].

[6] R. Monteiro, D. O'Connell and C.D. White, Black holes and the double copy, JHEP 12 (2014) 056 [arXiv: 1410.0239] [INSPIRE].

[7] Z. Bern, J.J. Carrasco, M. Chiodaroli, H. Johansson and R. Roiban, The Duality Between Color and Kinematics and its Applications, arXiv:1909.01358 [INSPIRE].

[8] Z. Bern et al., Ultraviolet Properties of $\mathcal{N}=8$ Supergravity at Five Loops, Phys. Rev. D 98 (2018) 086021 [arXiv: 1804.09311] [INSPIRE].

[9] Z. Bern, J.J.M. Carrasco and H. Johansson, New Relations for Gauge-Theory Amplitudes, Phys. Rev. D 78 (2008) 085011 [arXiv:0805.3993] [InSPIRE].

[10] M. Chiodaroli, Q. Jin and R. Roiban, Color/kinematics duality for general abelian orbifolds of $N=4$ super Yang-Mills theory, JHEP 01 (2014) 152 [arXiv:1311.3600] [INSPIRE].

[11] S.G. Naculich, Scattering equations and BCJ relations for gauge and gravitational amplitudes with massive scalar particles, JHEP 09 (2014) 029 [arXiv:1407.7836] [INSPIRE].

[12] H. Johansson and A. Ochirov, Double copy for massive quantum particles with spin, JHEP 09 (2019) 040 [arXiv: 1906.12292] [INSPIRE].

[13] M. Chiodaroli, M. Günaydin, H. Johansson and R. Roiban, Spontaneously Broken Yang-Mills-Einstein Supergravities as Double Copies, JHEP 06 (2017) 064 [arXiv: 1511.01740] [INSPIRE].

[14] M. Chiodaroli, M. Günaydin, H. Johansson and R. Roiban, Gauged Supergravities and Spontaneous Supersymmetry Breaking from the Double Copy Construction, Phys. Rev. Lett. 120 (2018) 171601 [arXiv:1710.08796] [INSPIRE].

[15] M. Chiodaroli, M. Günaydin, H. Johansson and R. Roiban, Non-Abelian gauged supergravities as double copies, JHEP 06 (2019) 099 [arXiv: 1812.10434] [INSPIRE].

[16] Y.F. Bautista and A. Guevara, On the Double Copy for Spinning Matter, arXiv:1908.11349 [INSPIRE].

[17] Y.F. Bautista and A. Guevara, From Scattering Amplitudes to Classical Physics: Universality, Double Copy and Soft Theorems, arXiv:1903.12419 [INSPIRE].

[18] D. Neill and I.Z. Rothstein, Classical Space-Times from the S Matrix, Nucl. Phys. B $\mathbf{8 7 7}$ (2013) 177 [arXiv:1304.7263] [INSPIRE].

[19] N.E.J. Bjerrum-Bohr, A. Cristofoli, P.H. Damgaard and H. Gomez, Scalar-Graviton Amplitudes, JHEP 11 (2019) 148 [arXiv:1908.09755] [INSPIRE]. 
[20] Z. Bern, C. Cheung, R. Roiban, C.-H. Shen, M.P. Solon and M. Zeng, Scattering Amplitudes and the Conservative Hamiltonian for Binary Systems at Third Post-Minkowskian Order, Phys. Rev. Lett. 122 (2019) 201603 [arXiv:1901.04424] [INSPIRE].

[21] F. Cachazo, S. He and E.Y. Yuan, Scattering of Massless Particles: Scalars, Gluons and Gravitons, JHEP 07 (2014) 033 [arXiv: 1309.0885] [INSPIRE].

[22] S. Mizera, Inverse of the String Theory KLT Kernel, JHEP 06 (2017) 084 [arXiv: 1610.04230] [INSPIRE].

[23] C. de Rham, G. Gabadadze and A.J. Tolley, Resummation of Massive Gravity, Phys. Rev. Lett. 106 (2011) 231101 [arXiv: 1011.1232] [INSPIRE].

[24] V. Del Duca, L.J. Dixon and F. Maltoni, New color decompositions for gauge amplitudes at tree and loop level, Nucl. Phys. B 571 (2000) 51 [hep-ph/9910563] [INSPIRE].

[25] A. Momeni, J. Rumbutis and A.J. Tolley, Massive Gravity from Double Copy, JHEP 12 (2020) 030 [arXiv: 2004.07853] [inSPIRE].

[26] R. Kleiss and H. Kuijf, Multi - Gluon Cross-sections and Five Jet Production at Hadron Colliders, Nucl. Phys. B 312 (1989) 616 [InSPIRE].

[27] G. Chen and Y.-J. Du, Amplitude Relations in Non-linear Sigma Model, JHEP 01 (2014) 061 [arXiv: 1311.1133] [INSPIRE].

[28] J.M. Cornwall, D.N. Levin and G. Tiktopoulos, Derivation of Gauge Invariance from High-Energy Unitarity Bounds on the s Matrix, Phys. Rev. D 10 (1974) 1145 [Erratum ibid. 11 (1975) 972] [INSPIRE].

[29] S. Weinberg, Phenomenological Lagrangians, Physica A 96 (1979) 327 [InSPIRE].

[30] C. Cheung, K. Kampf, J. Novotny and J. Trnka, Effective Field Theories from Soft Limits of Scattering Amplitudes, Phys. Rev. Lett. 114 (2015) 221602 [arXiv:1412.4095] [INSPIRE].

[31] G.R. Dvali, G. Gabadadze and M. Porrati, $4-D$ gravity on a brane in $5-D$ Minkowski space, Phys. Lett. B 485 (2000) 208 [hep-th/0005016] [INSPIRE].

[32] C. de Rham and G. Gabadadze, Selftuned Massive Spin-2, Phys. Lett. B 693 (2010) 334 [arXiv: 1006.4367] [INSPIRE].

[33] N. Arkani-Hamed, T.-C. Huang and Y.-t. Huang, Scattering Amplitudes For All Masses and Spins, arXiv:1709.04891 [INSPIRE].

[34] K. Hinterbichler, Theoretical Aspects of Massive Gravity, Rev. Mod. Phys. 84 (2012) 671 [arXiv: 1105.3735] [INSPIRE].

[35] S.F. Hassan and R.A. Rosen, Resolving the Ghost Problem in non-Linear Massive Gravity, Phys. Rev. Lett. 108 (2012) 041101 [arXiv:1106.3344] [InSPIRE].

[36] C. Cheung and G.N. Remmen, Positive Signs in Massive Gravity, JHEP 04 (2016) 002 [arXiv: 1601.04068] [INSPIRE].

[37] J.J. Bonifacio, Aspects of Massive Spin-2 Effective Field Theories, Ph.D. thesis, Oxford Uiversity, U.K. (2017).

[38] C. de Rham and G. Gabadadze, Generalization of the Fierz-Pauli Action, Phys. Rev. D 82 (2010) 044020 [arXiv: 1007.0443] [INSPIRE].

[39] J. Bonifacio and K. Hinterbichler, Bounds on Amplitudes in Effective Theories with Massive Spinning Particles, Phys. Rev. D 98 (2018) 045003 [arXiv: 1804.08686] [InSPIRE]. 
[40] C. De Rham, K. Hinterbichler and L.A. Johnson, On the (A)dS Decoupling Limits of Massive Gravity, JHEP 09 (2018) 154 [arXiv:1807.08754] [INSPIRE].

[41] N. Moynihan, Scattering Amplitudes and the Double Copy in Topologically Massive Theories, JHEP 12 (2020) 163 [arXiv:2006.15957] [INSPIRE].

[42] J. Bonifacio, K. Hinterbichler and R.A. Rosen, Constraints on a gravitational Higgs mechanism, Phys. Rev. D 100 (2019) 084017 [arXiv: 1903.09643] [InSPIRE].

[43] C. Cheung and C.-H. Shen, Symmetry for Flavor-Kinematics Duality from an Action, Phys. Rev. Lett. 118 (2017) 121601 [arXiv: 1612.00868] [INSPIRE].

[44] R. Monteiro and D. O'Connell, The Kinematic Algebra From the Self-Dual Sector, JHEP 07 (2011) 007 [arXiv:1105.2565] [INSPIRE].

[45] C. de Rham, Massive Gravity, Living Rev. Rel. 17 (2014) 7 [arXiv:1401.4173] [InSPIRE].

[46] H. van Dam and M.J.G. Veltman, Massive and massless Yang-Mills and gravitational fields, Nucl. Phys. B 22 (1970) 397 [InSPIRE].

[47] N. Arkani-Hamed, H. Georgi and M.D. Schwartz, Effective field theory for massive gravitons and gravity in theory space, Annals Phys. 305 (2003) 96 [hep-th/0210184] [INSPIRE]. 